\title{
Contribution to the knowledge of the family Sepsidae (Diptera) of Afrotropical Region
}

\section{K изучению двукрылых семейства Sepsidae (Diptera) Афротропической области}

\author{
A.L. Ozerov \\ A. $\Lambda$. Озеров \\ Zoological Museum, Lomonosov Moscow State University, Bol'shaya Nikitskaya 2, Moscow 125009, Russia. \\ E-mail: ozerov2455@rambler.ru \\ Зоологический музей, Московский государственный университет им. М.В. Ломоносова, Большая Никитская ул., 2, Москва \\ 125009 , Россия.
}

KEY WORDS: Diptera, Sepsidae, Afrotropical Region, new species, new records.

КЛЮЧЕВЫЕ СЛОВА: Diptera, Sepsidae, Афротропическая область, новые виды, новые данные по распространению.

ABSTACT. The results of the determination of Sepsidae materials from Afrotropical Region from collections of Czech University of Life Sciences Prague, Czech Republic, Tel Aviv University, Israel and The National Museum, Bloemfontein, Republic of South Africa are presented. 46 species from 13 genera were studied, gene-ral distributions for each species are provided. New records of the following species are given: Adriapontia freidbergi Ozerov, 2000 (Malawi); Afrosepsis camerounica Ozerov, 1996 (Togo); Brachythoracosepsis butikensis (Vanschuytbroeck, 1963) (Burundi, Malawi); B. freidbergi Ozerov, 1996 (Democratic Republic of the Congo); B. nodosa (Walker, 1849) (Togo); B. saothomensis Ozerov, 2000 (Democratic Republic of the Congo); Dicranosepsis takoensis (Vanschuytbroeck, 1963) (Benin, Togo); Diploosmeteriosepsis pilifemur (Munari, 1994) (Togo); Leptomerosepsis improvisa Ozerov, 1996 (Burundi); Paratoxopoda akuminambili Vanschuytbroeck, 1961 (Central African Republic); P. pilifemorata Soós, 1964 (Republic of South Africa); P. similis Ozerov, 1993 (Central African Republic, Nigeria, Togo); Saltella bezzii (Duda, 1926) (Central African Republic, Ethiopia); Sepsis arotrolabis Duda, 1926 (Malawi); Sepsis delectabilis Adams, 1905 (Burundi, Togo); S. dissimilis Brunetti, 1910 (Cameroon); S.s femoriseta Duda, 1926 (Cameroon); S. holaethiops Speiser, 1924 (Burundi); S. igniventris Thomson, 1869 (Benin, Burundi, Central African Republic); S. lateralis Wiedemann, 1830 (Benin, Burundi, Togo); S. luteola Ozerov, 2004 (Cameroon); S. oligochaeta Soós, 1962 (Cameroon); S. petulantis Adams, 1905 (Central African Republic, Togo); S. thoracica Robineau-Desvoidy, 1830 (Burundi, Cameroon, Central African Republic); Toxopoda bequaerti (Curran, 1929) (Burundi, Central African Re- public); T. nitida Macquart, 1851 (Cameroon); T. soror (Munari, 1994) (Central African Republic). In addition, four new species are described: Meroplius burundi sp.n. (Burundi), M. zimbabweensis sp.n. (Zimbabwe), Paratoxopoda mystacea sp.n. (Central African Republic), and Toxopoda haladai sp.n. (Central African Republic).

РЕЗЮМЕ. Представлены результаты определения материалов двукрылых семейства Sepsidae из Афротропического региона из колекций Чешского аграрного университета в Праге, Чехия, университета Тель-Авива, Израиль и Национального музея в Блумфонтейне, Южно-Африканская Республика. 41 вид из 13 родов были изучены, суммированы данные о распространении каждого вида. Приведены новые данные о распространении следующих видов: Adriapontia freidbergi Ozerov, 2000 (Малави); Afrosepsis camerounica Ozerov, 1996 (Того); Brachythoracosepsis butikensis (Vanschuytbroeck, 1963) (Бурунди, Малави); B. freidbergi Ozerov, 1996 (Демократическая республика Конго); B. nodosa (Walker, 1849) (Того); B. saothomensis Ozerov, 2000 (Демократическая республика Конго); Dicranosepsis takoensis (Vanschuytbroeck, 1963) (Бенин, Того); Diploosmeteriosepsis pilifemur (Munari, 1994) (Того); Leptomerosepsis improvisa Ozerov, 1996 (Бурунди); Paratoxopoda akuminambili Vanschuytbroeck, 1961 (Центральноафриканская Республика); P. pilifemorata Soós, 1964 (Южно-Африканская Республика); P. similis Ozerov, 1993 (Нигерия, Того, Центральноафриканская Республика); Saltella bezzii (Duda, 1926) (Центральноафриканская Республика, Эфиопия); Sepsis arotrolabis Duda, 1926 (Малави); S. delectabilis Adams, 1905 (Бурунди, Того); S. dissimilis Brunetti,

How to cite this article: Ozerov A.L. 2018. Contribution to the knowledge of the family Sepsidae (Diptera) of Afrotropical Region// Russian Entomol. J. Vol.27. No.3. P.323-335. doi: 10.15298/rusentj.27.3.15 
1910 (Камерун); S. femoriseta Duda, 1926 (Камерун); S. holaethiops Speiser, 1924 (Бурунди); S. igniventris Thomson, 1869 (Бенин, Бурунди, Центральноафриканская Республика); S. lateralis Wiedemann, 1830 (Бенин, Бурунди, Того); S. luteola Ozerov, 2004 (Камерун); S. oligochaeta Soós, 1962 (Камерун); S.s petulantis Adams, 1905 (Того, Центральноафриканская Республика); S. thoracica Robineau-Desvoidy, 1830 (Бурунди, Камерун, Центральноафриканская Республика); Toxopoda bequaerti (Curran, 1929) (Бурунди, Центральноафриканская Республика); T. nitida Macquart, 1851 (Камерун); T. soror (Munari, 1994) (Центральноафриканская Республика). В дополнение, четыре новых для науки вида описаны: Meroplius burundi sp.n. (Бурунди), Meroplius zimbabweensis sp.n. (Зимбабве), Paratoxopoda mystacea sp.n. (Центральноафриканская Республика) и Toxopoda haladai sp.n. (Центральноафриканская Республика).

\section{Introduction}

Sepsidae are small- or medium-sized (body length: 2-12 mm) black, rarely grey, or yellow acalyptrate flies, many of which superficially resemble winged ants, and are easily distinguished from other families, except the Neotropical family Rhopalomeridae, by the presence of one or more setae situated below the posterior thoracic spiracle.

More than 120 species in 18 genera occur in the Afrotropical Region [Ozerov, 2005, 2006, 2010, 2011], but the sepsid fauna of the majority of African countries has remained completely unknown till now.

The present work is based on the materials of Sepsidae from Czech University of Life Sciences Prague (CULS), Tel Aviv University, Israel (TAU) and The National Museum, Bloemfontein, Republic of South Africa (NMB). This paper represents the additional contribution to the study of the Afrotropical Sepsidae fauna. 46 species from 13 genera were studied, 27 species are recorded firstly for some countries. The terminology used for the description of new species follows McAlpine [1981], Cumming et al. [2009], and Stuckenberg [1999].

The following abbreviations are used: RSA - Republic of South Africa, D.R. Congo - Democratic Republic of the Congo; ZMUM - Zoological Museum, Moscow State University, Moscow, Russia.

\section{Taxonomic part}

\section{Adriapontia capensis (Hennig, 1960)}

MATERIAL. RSA, Eastern Cape, Hogsback, Never Daunted, 32 $35.725^{\prime}$ 'S 26⒌873'E, 28-29.III.2011, 1138 m, A.H. KirkSpriggs, malaise traps, indigenous Afromontane forest $\left(3 \sigma^{\top} \sigma^{\top}, 2\right.$ 90 , NMB); Eastern Cape, Hogsback, Redwood trail, 32 35.337's 265ㄷ.135'E, 26.III.2011, 1169 m, A.H. Kirk-Spriggs, sweeping forest paths and glades $(1+\mathrm{O}, \mathrm{NMB})$; Western Cape, Keurbos forest, $33^{\circ} 54.435^{\prime}$ S 234 $2.714^{\prime}$ E, 28-30.III.2009, 500 m, A.H. KirkSpriggs \& S. Otto, sweeping indigenous montane forest $\left(4 \sigma^{7} \sigma^{7}, 5\right.$ + , , NMB); KZN, Royal Natal N.P., Thendele, $28^{\circ} 42.378^{\prime} \mathrm{S} 28^{\circ}$ 56.083'E, 15-17.II.2010, 1600 m, A.H. Kirk-Spriggs, malaise traps, Leucosedea dominated scrub (3 $\circ \circ$, NMB).

DISTRIBUTION. Republic of South Africa.

\section{Adriapontia freidbergi Ozerov, 2000}

MATERIAL. Malawi, Zomba Plateau Trout Farm, $15^{\circ} 21.4^{\prime} \mathrm{S}$ $35^{\circ} 18.1^{\prime} \mathrm{E}, 1477 \mathrm{~m}, 5$ and 10.I.2010, A. Freidberg (8 $\mathrm{O}^{\top} \mathrm{O}^{\top}$, TAU, ZMUM); Nyika National Park, Zovo Chipolo Forest, 10³4.5'S

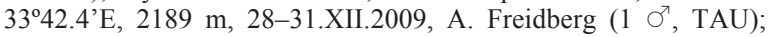
Viphya Mts., Chikangawa, $11^{\circ} 50.5^{\prime} \mathrm{S} 33^{\circ} 48.2^{\prime} \mathrm{E}, 1707 \mathrm{~m}$, 26.XII.2009, A. Freidberg (1 $\sigma^{\top}$, TAU).

DISTRIBUTION. Malawi (first record), Tanzania.

Afromeroplius semlikiensis (Vanschuytbroeck, 1963)

MATERIAL. Kenya, Rift Valley Prov., Mpala Research Station at: $00^{\circ} 17.635^{\prime} \mathrm{S} 36^{\circ} 53.947^{\prime} \mathrm{E}, 17-18 . \mathrm{IV} .2011,1792 \mathrm{~m}$, A.H. \& M.K. Kirk-Spriggs, malaise traps, dry Acacia savanna $\left(2 \sigma^{7} \sigma^{7}\right.$, NMB).

DISTRIBUTION. Democratic Republic of the Congo, Kenya, Namibia.

Afrosepsis camerounica Ozerov, 1996

MATERIAL. Togo, Kloto waterfall, 6 6 57' N $0^{\circ} 36^{\prime} \mathrm{E}, 336 \mathrm{~m}$, 22.I.2011, A. Freidberg (1 $0^{7}$, TAU).

DISTRIBUTION. Cameroon, Togo (first record).

Australosepsis niveipennis (Becker, 1903)

MATERIAL. Cameroon, Far-North Reg., Mayo-Tsanga, Mogode-Kama, $10^{\circ} 32.598^{\prime} \mathrm{N} 13^{\circ} 35.027^{\prime} \mathrm{E}, 7$.VIII.2013, 1056 m, A.H. Kirk-Spriggs, sweeping domestic donkey dung ( $1 \Im^{7}, 1$, NMB); North Reg., Vina, Tchabal village at: (1) $07^{\circ} 36.803^{\prime} \mathrm{N} 13^{\circ}$ 32.622'E, 12.VIII.2013, 1270 m, A.H. Kirk-Spriggs, sweeping in deep shade (1 9 , NMB); North Reg., Mayo-Louti, Bossom at: (2) $09^{\circ} 55.233^{\prime} \mathrm{N} 13^{\circ} 46.645^{\prime} \mathrm{E}, 6$.VIII.2013, 475 m, A.H. Kirk-Spriggs, sweeping understory vegetation in deep shade ( 1 , , NMB); Ethiopia, $45 \mathrm{~km}$ NE Arba Minch, $6^{\circ} 17^{\prime} \mathrm{N} 37^{\circ} 47^{\prime} \mathrm{E}, 1200 \mathrm{~m}, 10 . \mathrm{V} .2015$, J. Halada ( 1 +, CULS); Kenya, Mt. Kenya, Castle Forest, $0^{\circ} 25^{\prime} \mathrm{S}$ $37^{\circ} 19^{\prime} \mathrm{E}, 1920 \mathrm{~m}, 12 . I I .2011$, A. Freidberg (1 フ', TAU); Gilgil,

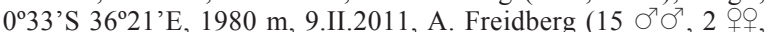
TAU); Rift Valley Prov., Mpala Research Station at: $00^{\circ} 17.635^{\prime} \mathrm{S}$ 3653.947'E, 17-18.IV.2011, 1792 m, A.H. \& M.K. Kirk-Spriggs, malaise traps, dry Acacia savanna (1 $\sigma^{7}, 1$, NMB); Malawi, Salima, 10 km E, Rt.S122, 13²46.1'S 34'33.2'E, 494 m, 4.I.2010, on acacia, A. Freidberg (2 $\sigma^{\top} \sigma^{\top}$, TAU); Lake Chilwa, Kachulu, Rt. S143, 15²2.3'S 3535.4'E, 657 m, 6.I.2010, A. Freidberg (1 $\sigma^{7}$, TAU); Namibia, Katima Mulilo Dist., Mavunje campsite at: $17^{\circ} 55.141^{\prime} \mathrm{S} 23^{\circ} 19.073^{\prime} \mathrm{E}, 22-26 . X I .2012,945$ m, A.H. KirkSpriggs, malaise traps, Kwando River floodplain (2 $90, \mathrm{NMB}$ ); prov. Erongo, $28 \mathrm{~km} \mathrm{~S}$ of Karibib, $22^{\circ} 11.64 \mathrm{~S} 15^{\circ} 51.24 \mathrm{E}, 1150 \mathrm{~m}$, 28.III.2017, J. Halada (1 $\sigma^{7}$, CULS); Nige-ria, Gashaka Gumfi NP, $30 \mathrm{~km}$ SE Senti, $7^{\circ} 21^{\prime} \mathrm{N} 1^{\circ} 32^{\prime}$ 'E, 380-560 m, 24.IV.8.V.2011, J. Halada ( $2 \sigma^{\top} \sigma^{\top}, 1$, CULS); 28 km SEE Abuja, $09^{\circ}$

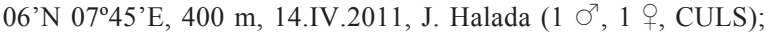
Togo, Kloto, Kouma Tokoli, 6 6 $58^{\prime} \mathrm{N} 0^{\circ} 35^{\prime} \mathrm{E}, 530$ m, 20.I.2011, A. Freidberg $(1+$, TAU).

DISTRIBUTION. Angola, Botswana, Cameroon, Ethiopia, Ghana, Kenya, Madagascar, Malawi, Namibia, Nigeria, Republic of the Congo, Republic of South Africa, Sierra Leone, Sudan, Swaziland, Tanzania, Togo, Uganda, Zimbabwe. - Australasian/Oceanian, Oriental, Palaearctic. 1963)

Brachythoracosepsis butikensis (Vanschuytbroeck,

MATERIAL. Burundi, Bururi Province, Bururi Nat. Res. cloud

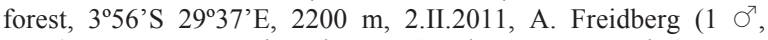
TAU); Malawi, Zomba Plateau, South Water Board Cottage, 
$15^{\circ} 21.5^{\prime} \mathrm{S} 35^{\circ} 18.3^{\prime} \mathrm{E}, 1471 \mathrm{~m}$, 5.I.2010, A. Freidberg ( $\sigma^{\gamma}, 1$ ㅇ, TAU); Zomba Plateau Trout Farm, 1521.4'S 35¹8.1'E, 1477 m, 10.I.2010, A. Freidberg (1 $\sigma^{7}$, TAU); Kenya, Central Prov., Katura forest, Nairobi at: $01^{\circ} 14.504$ 'S $36^{\circ} 49.452^{\prime} \mathrm{E}, 23 . I V .2011,1720 \mathrm{~m}$, A.H. Kirk-Spriggs, sweeping in shaded mixed upland indigenous forest (1 $\left.\sigma^{7}, \mathrm{NMB}\right)$.

DISTRIBUTION. Burundi (first record), Cameroon, Democratic Republic of the Congo, Ethiopia, Ghana, Malawi (first record), Kenya, Nigeria, Republic of South Africa, Tanzania, Zambia, Zimbabwe.

\section{Brachythoracosepsis freidbergi Ozerov, 1996}

MATERIAL. D.R. Congo, Oriental Province, Likombo forest, $2 \mathrm{~km}$ SW Bomane, $1.28349^{\circ} \mathrm{N} 23.72358^{\circ} \mathrm{E}, 20-22 . V .2010$, A.H Kirk-Spriggs, malaise traps, lowland evergreen primary forest (disturbed), Boyekoli Ebale Congo Expedition 2010 (1 ア $\Im^{\top}$ NMB); Oriental Province, Yafira forest transect, $00.69594^{\circ} \mathrm{N} 24.20107^{\circ} \mathrm{E}$, 29-31.V.2010, A.H. Kirk-Spriggs, malaise traps, lowland evergreen swamp forest, Boyekoli Ebale Congo Expedition 2010 (1 $\sigma^{7}$, $\mathrm{NMB}$ ); Oriental Province, Yafira forest transect, $00.70216^{\circ} \mathrm{N}$ 24.20060 ${ }^{\circ}$, 29-31.V.2010, A.H. Kirk-Spriggs, malaise traps, secondary lowland evergreen forest, Boyekoli Ebale Congo Expedition 2010 (1 $\left.\sigma^{7}, \mathrm{NMB}\right)$.

DISTRIBUTION. Democratic Republic of the Congo (first record), Cameroon, Kenya.

\section{Brachythoracosepsis nodosa (Walker, 1849)}

MATERIAL. Cameroon, Northwest Reg., Mezam, Bafut village at: $06^{\circ} 05.026^{\prime} \mathrm{N} 10^{\circ} 07.442^{\prime} \mathrm{E}, 17-19$.VIII.2013, $1060 \mathrm{~m}, \mathrm{~A} . \mathrm{H}$ Kirk-Spriggs, malaise traps, cultivated plots \& degraded forest (1 +, NMB); D.R. Congo, Oriental Province, Bomane village area at: $01^{\circ} 16283^{\prime} \mathrm{N} 23^{\circ} 43.994^{\prime} \mathrm{E}, 20-24 . V .2010$, A.H. Kirk-Spriggs, sweeping bush paths \& village environs, Boyekoli Ebale Congo Expedition 2010 ( $1 \sigma^{\top}, 3$ of , NMB); Oriental Province, Bomane village area at: $01^{\circ} 16283^{\prime} \mathrm{N} 23^{\circ} 43.994^{\prime} \mathrm{E}, 20-24$.V.2010, A.H. KirkSpriggs, malaise traps, lowland evergreen secondary forest, Boyekoli Ebale Congo Expedition 2010 (1 + , NMB); Oriental Province, Likombo forest, $2 \mathrm{~km} \mathrm{SW}$ Bomane, $1.28349^{\circ} \mathrm{N} 23.72358^{\circ} \mathrm{E}, 20$ 22.V.2010, A.H. Kirk-Spriggs, malaise traps, lowland evergreen primary forest (disturbed), Boyekoli Ebale Congo Expedition 2010 (1 9 , NMB); Malawi, Zomba Plateau Trout Farm, $15^{\circ} 21.4^{\prime} \mathrm{S}$ $35^{\circ} 18.1^{\prime} \mathrm{E}, 1477 \mathrm{~m}, 10 . \mathrm{I} .2010$, A. Freidberg (1 + TAU); Togo, Kloto Agome-Yoh, 6º57'N 0³6'E, 333 m, 21.I.2011, A. Freidberg (1 + , TAU).

DISTRIBUTION. Cameroon, Comoros, Democratic Republic of the Congo, Ethiopia, Guinea, Ivory Coast, Kenya, Madagascar, Malawi, Nigeria, Republic of South Africa, São Tomé and Príncipe, Sierra Leone, Tanzania, Togo (first record), Uganda.

Brachythoracosepsis ruanoliensis (Vanschuytbroeck, 1963)

MATERIAL. D.R. Congo, Oriental Province, Bomane village area at: $01^{\circ} 16283^{\prime} \mathrm{N} 23^{\circ} 43.994^{\prime} \mathrm{E}, 20-24$. V.2010, A.H. KirkSpriggs, malaise traps, lowland evergreen secondary forest, Boyekoli Ebale Congo Expedition 2010 (1 $\sigma^{\top}, 1$, , NMB); Oriental Province, Bomane village area at: $01^{\circ} 16283^{\prime} \mathrm{N} 23^{\circ} 43.994^{\prime} \mathrm{E}, 20$ 24.V.2010, A.H. Kirk-Spriggs, sweeping bush paths \& village environs, Boyekoli Ebale Congo Expedition 2010 (1 +, NMB); Oriental Province, Likombo forest, $2 \mathrm{~km} \mathrm{SW}$ Bomane, $1.28349^{\circ} \mathrm{N}$ $23.72358^{\circ}$ E, 20-22.V.2010, A.H. Kirk-Spriggs, malaise traps, lowland evergreen primary forest (disturbed), Boyekoli Ebale Congo Expedition 2010 ( 1 \%, 2 Oᄋ, NMB); Oriental Province, Yafira forest transect, $00.70216^{\circ} \mathrm{N} 24.20060^{\circ} \mathrm{E}, 29-31 . V .2010$, A.H. KirkSpriggs, malaise traps, secondary lowland evergreen forest, Boyekoli Ebale Congo Expedition 2010 (2 $\sigma^{7} \sigma^{7}, 1$ ㅇ, NMB); Oriental
Province, Lieki village, $1 \mathrm{~km} \mathrm{~S}$ at: $00^{\circ} 41.483^{\prime} \mathrm{N} 24^{\circ} 14.271^{\prime} \mathrm{E}, 27-$ 28.V.2010, A.H. Kirk-Spriggs, hanging trap, bailed feces secondary forest, Boyekoli Ebale Congo Expedition 2010 (1 9 , NMB); Oriental Province, Eyolo forest, ca $2 \mathrm{~km}$ E Lieki, $0.69642^{\circ} \mathrm{N} 24$. 24186 ${ }^{\circ}$ E, 25-29.V.2010, A.H. Kirk-Spriggs, malaise traps, lowland evergreen swamp forest, Boyekoli Ebale Congo Expedition 2010 ( $6 \sigma^{7} \sigma^{7}, 3$ 우, NMB); Oriental Province, Yafira forest transect, $00.70269^{\circ} \mathrm{N} 24.20080^{\circ} \mathrm{E}, 29-31 . V .2010$, A.H. Kirk-Spriggs, malaise traps, primary lowland evergreen forest, Boyekoli Ebale Congo Expedition 2010 ( $1 \sigma^{7}$, NMB); Oriental Province, Yafira forest transect, $00.69594^{\circ} \mathrm{N} 24.20107^{\circ} \mathrm{E}, 29-31 . V .2010$, A.H. KirkSpriggs, malaise traps, lowland evergreen swamp forest, Boyekoli Ebale Congo Expedition 2010 (1 $\left.\sigma^{7}, \mathrm{NMB}\right)$.

DISTRIBUTION. Cameroon, Democratic Republic of the Congo, Kenya, Nigeria, Sierra Leone, Uganda.

\section{Brachythoracosepsis saothomensis Ozerov, 2000}

MATERIAL. D.R. Congo, Oriental Province, Yafira forest transect, 00.70269N 24.20080E, 29-31.V.2010, A.H. Kirk-Spriggs, malaise traps, primary lowland evergreen forest, Boyekoli Ebale Congo Expedition 2010 (1 $\left.\sigma^{7}, \mathrm{NMB}\right)$;

DISTRIBUTION. Democratic Republic of the Congo (first record), São Tomé and Príncipe.

Dicranosepsis takoensis (Vanschuytbroeck, 1963)

MATERIAL. Benin, Amanhoue, Gohome, Djakotomey, 6 ${ }^{\circ} 50^{\prime} \mathrm{N}$ $1^{\circ} 46^{\prime} \mathrm{E}, 53 \mathrm{~m}, 15 . \mathrm{I} .2011$, A. Freidberg (7 $\sigma^{7} \mathrm{\sigma}^{7}, 1$ ㅇ, TAU); Cameroon, Northwest Reg., Menoua, Université Dschang, 05²6.761'N $10^{\circ} 04.237^{\prime} \mathrm{E}, 16 . \mathrm{VIII} .2013,1402$ m, A.H. Kirk-Spriggs, sweeping cultivated plots \& banana groves $\left(2 \sigma^{7} \sigma^{7}, 1 \circ\right.$, NMB); D.R. Congo, Oriental Province, Lieki village area at: $00^{\circ} 41.117^{\prime} \mathrm{N} 24^{\circ} 14.362^{\prime} \mathrm{E}$, 25.V.-4.VI.2010, A.H. Kirk-Spriggs, malaise traps, bush paths \& village environs, Boyekoli Ebale Congo Expedition 2010 (1 + NMB); Oriental Province, Bomane village area at: $01^{\circ} 16283^{\prime} \mathrm{N} 23^{\circ}$ 43.994'E, 20-24.V.2010, A.H. Kirk-Spriggs, sweeping bush paths \& village environs, Boyekoli Ebale Congo Expedition 2010 (1 + NMB); Togo, Kloto, Kouma Konda, 6 ${ }^{\circ} 57^{\prime} \mathrm{N} 0^{\circ} 35^{\prime} \mathrm{E}, 620$ m, 2022.I.2011, A. Freidberg (4 $\sigma^{7} \sigma^{7}$, TAU).

DISTRIBUTION. Angola, Benin (first record), Cameroon, Comoros, Democratic Republic of the Congo, Ethiopia, Guinea, Kenya, Nigeria, Republic of the Congo, Togo (first record), Uganda.

\section{Diploosmeteriosepsis pilifemur (Munari, 1994)}

MATERIAL. Togo, Kloto, Kouma Tokoli, 6 ${ }^{\circ} 58^{\prime} \mathrm{N} 0^{\circ} 35^{\prime} \mathrm{E}, 530$ m, 20.I.2011, A. Freidberg (1 +, TAU)

DISTRIBUTION. Nigeria, Sierra Leone, Togo (first record).

\section{Leptomerosepsis improvisa Ozerov, 1996}

MATERIAL. Burundi, Bururi Province, Siguvuyaye gallery forest, 3⒌'S 293' $\mathrm{E}, 1790 \mathrm{~m}$, 2.II.2011, A. Freidberg (2 $\sigma^{7} \sigma^{7}$, TAU); Cameroon, North Reg., Vina, Tchabal village at: (1) $07^{\circ} 36.803^{\prime} \mathrm{N} 13^{\circ} 32.622^{\prime} \mathrm{E}, 12$.VIII.2013, 1270 m, A.H. KirkSpriggs, sweeping in deep shade (7 $9+, \mathrm{NMB})$.

DISTRIBUTION. Burundi (first record), Cameroon, Kenya, Uganda.

\section{Leptomerosepsis simplicicrus (Duda, 1926)}

MATERIAL. Cameroon, Northwest Reg., Menoua, Université Dschang, 0526.761'N 1004.237'E, 16.VIII.2013, $1402 \mathrm{~m}$, A.H. Kirk-Spriggs, sweeping cultivated plots \& banana groves (1 +, NMB); D.R. Congo, Oriental Province, Bomane village area at: 
$01^{\circ} 16283^{\prime} \mathrm{N} 23^{\circ} 43.994^{\prime} \mathrm{E}, 20-24 . V .2010$, A.H. Kirk-Spriggs, malaise traps, lowland evergreen secondary forest, Boyekoli Ebale Congo Expedition 2010 (1 9 , NMB).

DISTRIBUTION. Cameroon, Democratic Republic of the Congo, Ethiopia, Kenia, Nigeria, Uganda.

\section{Meroplius burundi sp.n.} Figs 1-4.

MATERIAL. Holotype $\sigma^{7}$, Burundi, Kayanza Prov., Park National de la Kabira, Rwegura Sector, 0255.320'S 29³0.067'E, 21-26.XI.2010, 2237 m, A.H. Kirk-Spriggs, malaise traps, indigenous Afromontane forest (NMB).

DESCRIPTION. MALE. Body length $4.5 \mathrm{~mm}$; wing length $4.2 \mathrm{~mm}$. Head and eye in lateral view roundish. Frons black, subshining. Facial carina yellowish; antennal grooves blackish. Gena black; height of gena below eye approximately $1 / 7$ times as long as vertical diameter of eye. Clypeus black. Postcranium black, delicately greyish dusted. 1 small orbital, 1 ocellar, 1 divergent postocellar, 1 inner vertical, 1 outer vertical setae present. Antenna yellowish, but postredicel darkened from outside. Postpedicel about 1.5 times longer than wide. One small vibrissa present.

Thorax black. Scutum delicately greyish dusted; 1 postpronotal, 2 notopleural, $0+1$ supra-alar, 1 postalar, $0+1$ dorsocentral setae present. Proepisternum greyish dusted. Proepimeron and anepisternum shining. Katepisternum shining, with densely greyish dusted stripe along upper margin. Anepimeron, katepimeron and metepimeron shining. Meron, metepisternum, katatergite and anatergite delicately greyish dusted. Mediotergite delicately greyish dusted, but shining under scutellum. Postmetacoxal bridge absent. Scutellum greyish dusted, with a pair of long apical setae and a pair of basal setulae.

Legs. Fore coxa and tibia yellow, fore femur darkened, mid and hind femora black, but yellow basally, mid and hind tibiae black, all tarsi blackish, only tarsomere 1 of fore tarsus yellowish. Fore femur and tibia as in Fig. 1. Mid coxa bare. Mid tibia without setae, except apicals. Hind tibia with good visible osmeterium in basal half anterodorsally and with small preapical dorsal seta.

Wing clear, only basal-costal cell darkened; veins brown. Calypters whitish; margin of upper calypter white, margin of lower calypter darkened. Halter whitish. Alula entirely covered with microtrichia.

Abdomen black, shining. Sternite 4 as in Fig. 2. Surstyli stick-like, curved basally (Fig. 4).

FEMALE unknown.

COMPARISON. The new species is close to Meroplius cordylophorus Hennig, 1954 by structure of foreleg (Fig. 9) and by structure of male surstyli, but surstyli of $M$. cordylophorus are broadening apically and with more or less long hairs at apex (Fig. 10).

DISTRIBUTION. Burundi.

\section{Meroplius zimbabweensis sp.n.} Figs 5-8.

MATERIAL. Holotype $\sigma^{7}$, Zimbabwe, $60 \mathrm{~km}$ NNW Bulawayo, 1941'N 28 21'E, 1200 m, 18-19.XII.2011, J. Halada (CULS). Paratypes $5 \bigcirc^{7} \sigma^{7}$, same labels as holotype (CULS, ZMUM).
DESCRIPTION. MALE. Body length 2.5-3.1 mm; wing length $2.0-2.5 \mathrm{~mm}$. Head in lateral view slightly flattened dorsoventrally. Eye in lateral view roundish. Frons black, subshining. Facial carina brown; antennal grooves brown or blackish. Gena black; height of gena below eye approximately 1/5 times as long as vertical diameter of eye. Clypeus black. Postcranium black, greyish dusted. 1 small orbital, 1 ocellar, 1 divergent postocellar, 1 inner vertical, 1 outer vertical setae present. Antenna from yellow to dark brown. Postpedicel in profile oval, slightly longer than wide. One small vibrissa present.

Thorax black. Scutum delicately greyish dusted; 1 postpronotal, 2 notopleural, $0+1$ supra-alar, 1 postalar, $0+1$ dorsocentral setae present. Proepisternum greyish dusted. Proepimeron and anepisternum shining. Katepisternum shining, with densely greyish dusted stripe along upper margin. Anepimeron, katepimeron, meron, metepisternum, and metepimeron shining. Katatergite and anatergite delicately greyish dusted. Mediotergite greyish dusted, but shining under scutellum. Postmetacoxal bridge absent. Scutellum delicately greyish dusted, with a pair of long apical setae and a pair of basal setulae.

Legs. Fore coxa, fore femur and tibia yellow, mid femur and tibia black, hind femur black, but yellow basally, hind tibia black, all tarsi yellow in ground colour, but tarsomeres 3-5 blackish. Fore femur and tibia as in Fig. 5. Mid coxa bare. Mid tibia with one anteroventral seta in apical third, also with a ring of apical setae. Hind tibia with good visible osmeterium in basal half anterodorsally and with short preapical dorsal seta.

Wing clear, only basal-costal cell darkened; veins brown. Calypters whitish; margin of upper calypter white, margin of lower calypter darkened. Halter whitish. Alula entirely covered with microtrichia.

Abdomen black, shining. Sternites 4 and 5 as in Fig. 6. Surstyli short, broaded apically (Figs 7, 8).

FEMALE unknown.

COMPARISON. The new species is close to Meroplius zambianus Ozerov, 2011 by structure of male surstyli, but surstyli of $M$. zimbabweensis is shorter, not broadening medially inside as in Figs 12 and 13, but broadening apically (Fig. 8). The new species differs also by the structure of male sternite 4 (Fig. 6 and Fig. 11).

DISTRIBUTION. Zimbabwe.

Paratoxopoda akuminambili Vanschuytbroeck, 1961

MATERIAL. Central African Republic, $60 \mathrm{~km}$ W Bouar,

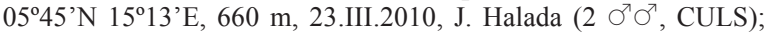
$60 \mathrm{~km} \mathrm{~W}$ Bouar, $05^{\circ} 42^{\prime} \mathrm{N} 16^{\circ} 00^{\prime} \mathrm{E}, 860 \mathrm{~m}$, 30.III.2010, J. Halada (2 Ơ $\sigma^{7}$, CULS).

DISTRIBUTION. Central African Republic (first record), Democratic Republic of the Congo, Ethiopia.

Paratoxopoda amonane Vanschuytbroeck, 1961

MATERIAL. RSA, KZN, Ndumo Game R. at: $26^{\circ} 53.597^{\prime} \mathrm{S}$ $32^{\circ} 12.559^{\prime} \mathrm{E}, 27 . X I .2009$, A.H. Kirk-Spriggs, ex dung of Cape Buffalo (Syncerus caffer) (4 $\left.\bigcirc^{\top} \sigma^{7}, \mathrm{NMB}\right)$. 


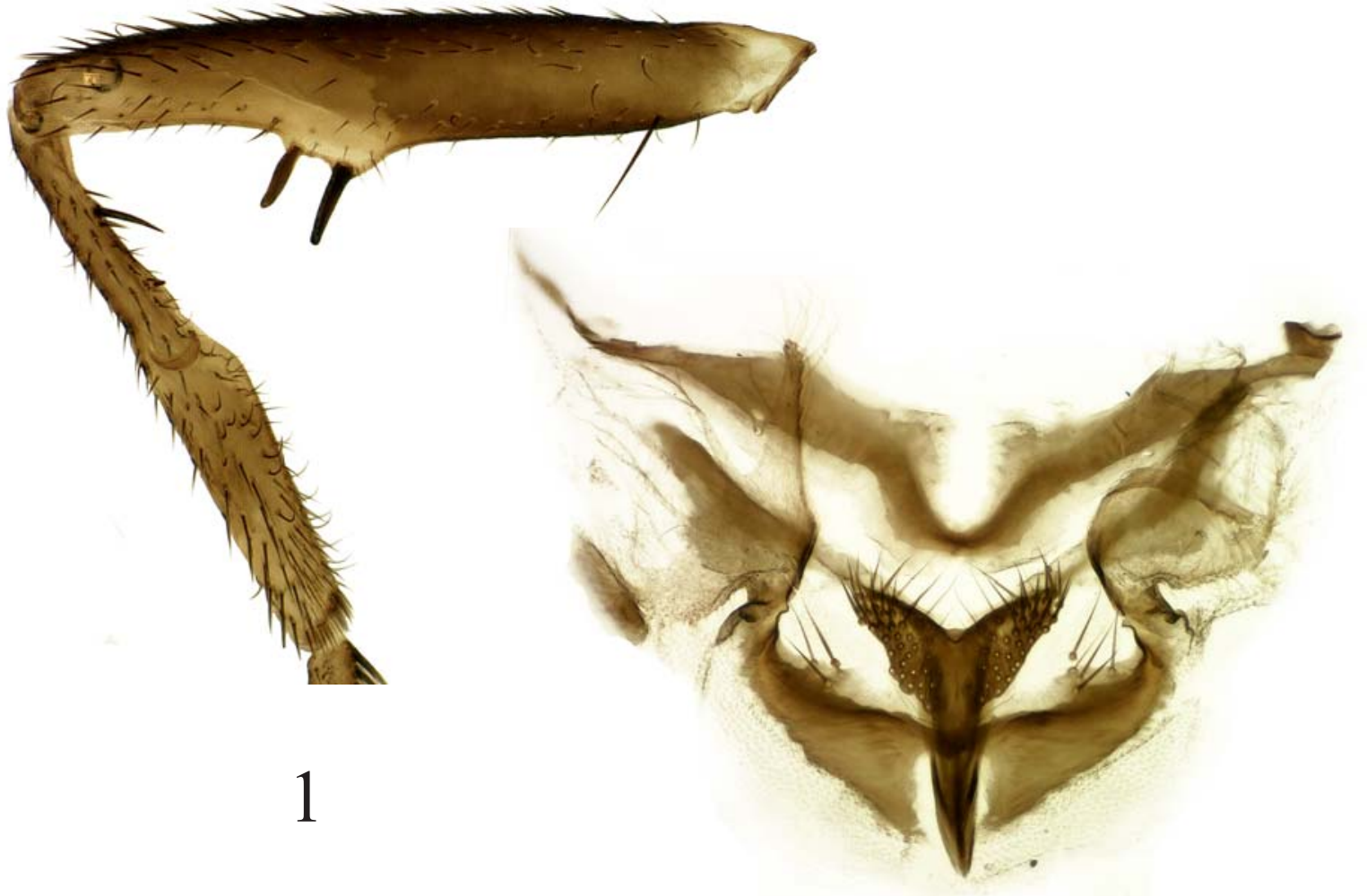

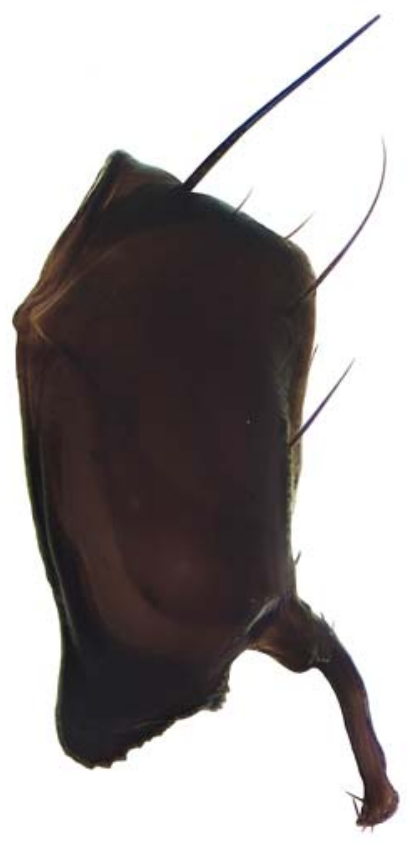

3

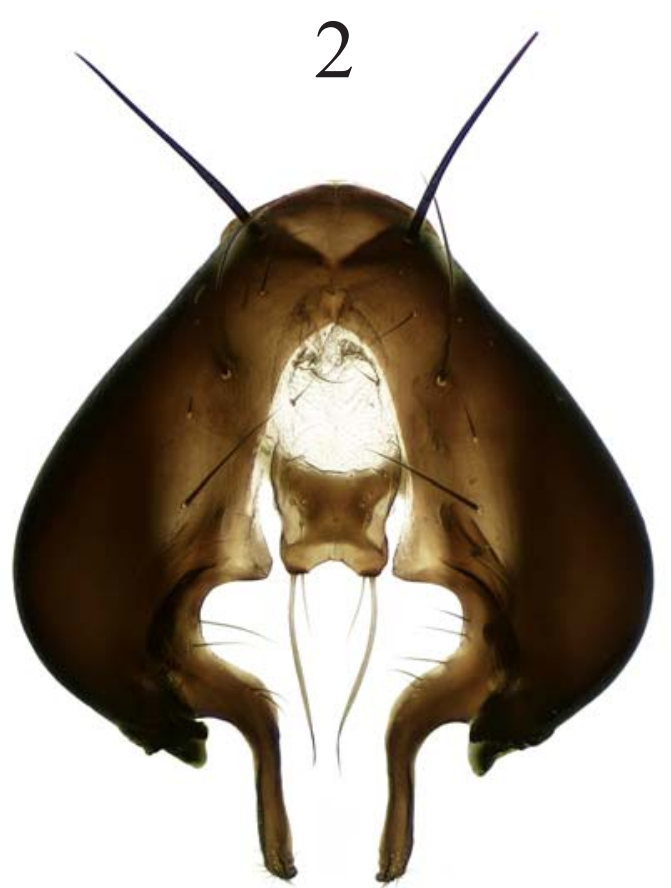

4

Figs. 1-4. Meroplius burundi sp.n., $\sigma^{7}$ holotype: 1 - fore femur and tibia, anteriorly; 2 - sternite 4; 3 - epandrium, cerci and surstyli, lateral view; 4 - same, dorsal view.

Рис. 1-4. Meroplius burundi sp.n., Ơ голотип: 1 - бедро и голень передней ноги, спереди; 2 - стернит 4; 3 - эпандрий, церки и сурстили, сбоку; 4 - то же, сверху. 


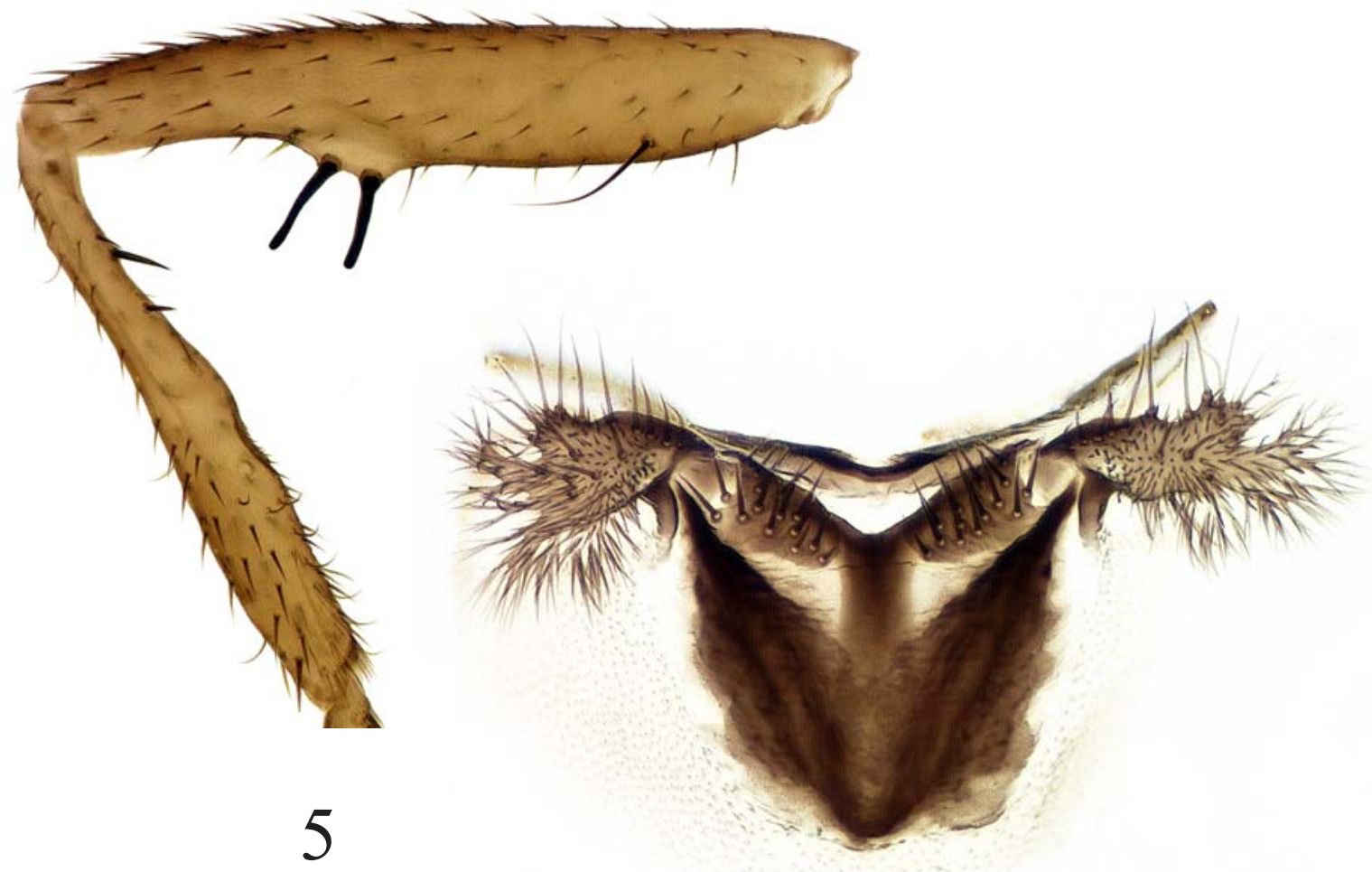

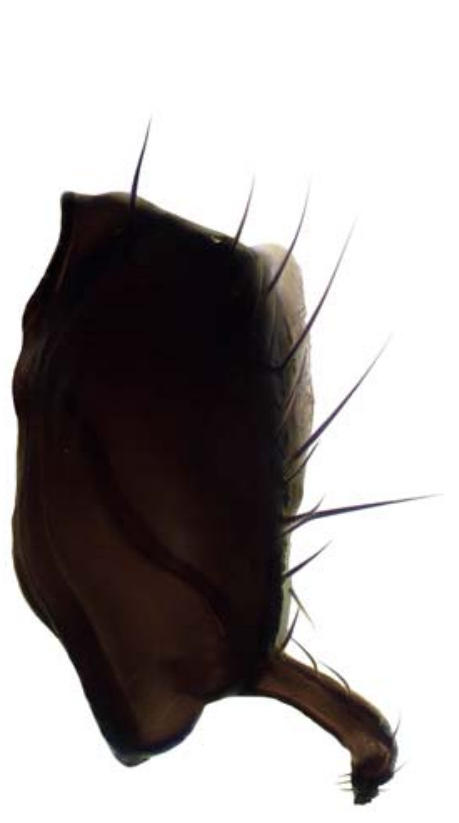

7

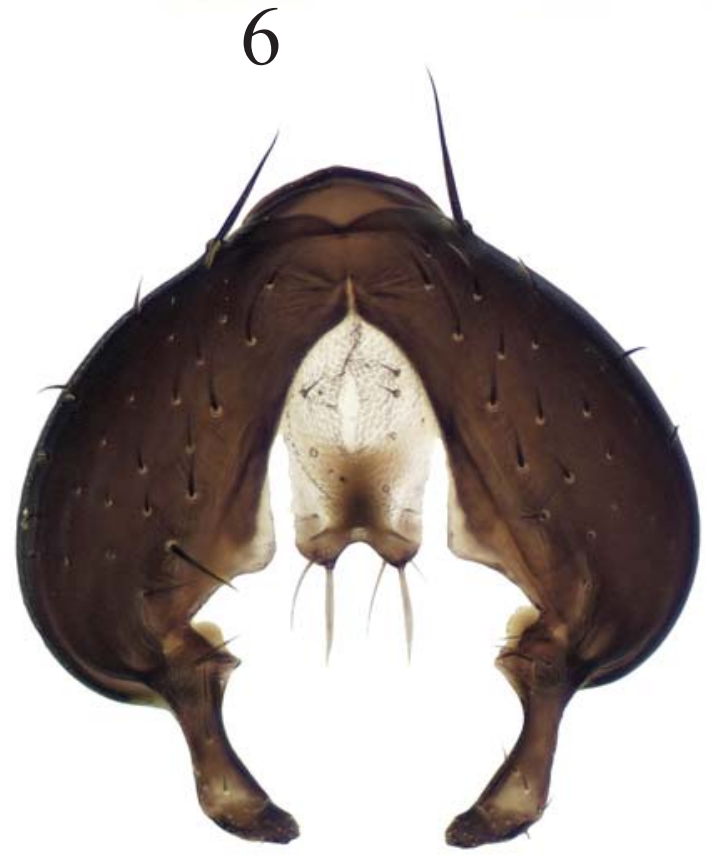

8

Figs. 5-8. Meroplius zimbabweensis sp.n., $\sigma^{7}$ paratype: 5 - fore femur and tibia, anteriorly; 6 - sternite 4; 7 - epandrium, cerci and surstyli, lateral view; 8 - same, dorsal view.

Рис. 5-8. Meroplius zimbabweensis sp.n., O $^{7}$ паратип: 5 - бедро и голень передней ноги, спереди; 6 - стернит 4; 7 эпандрий, церки и сурстили, сбоку; 8 - то же, сверху. 

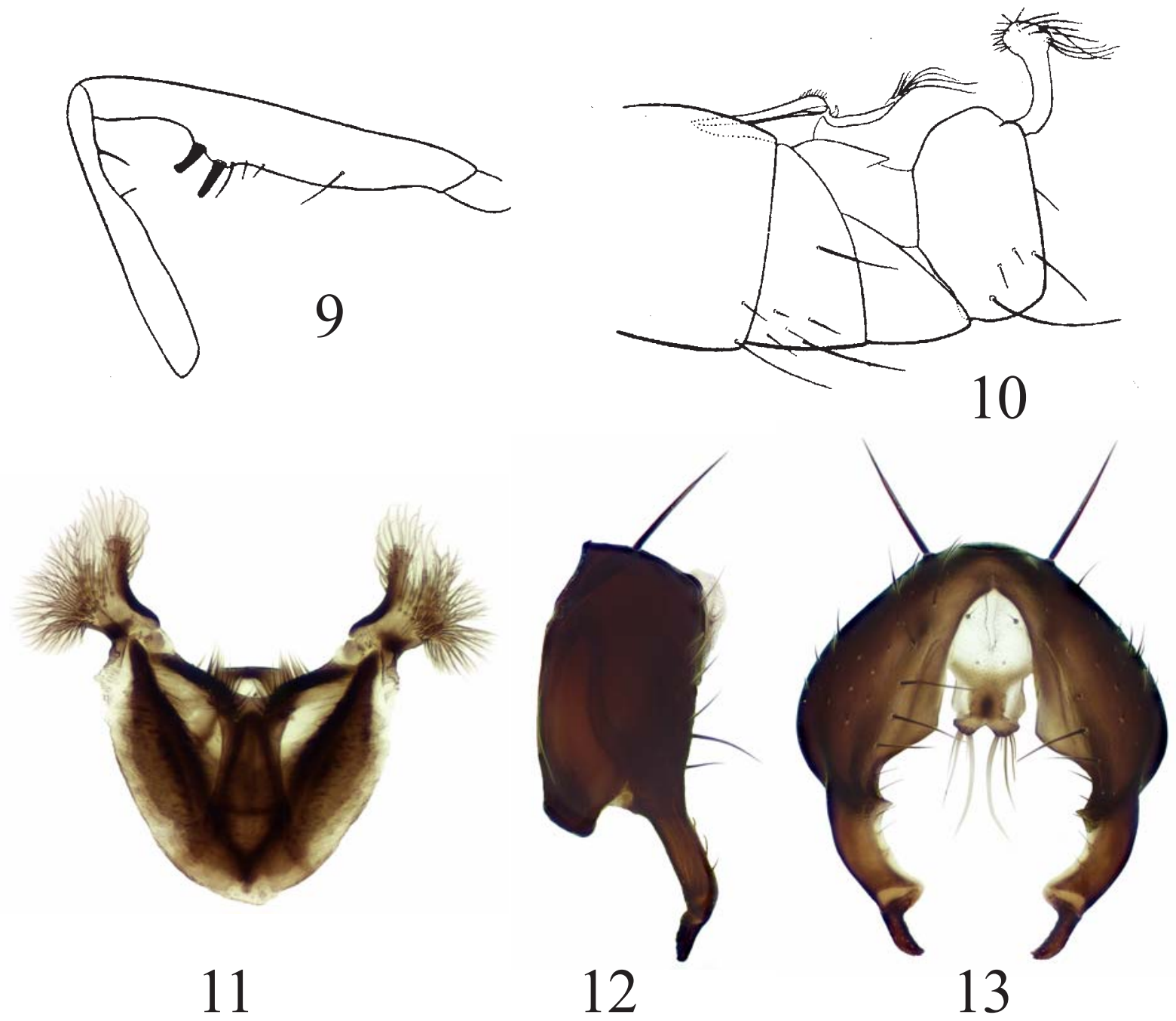

Figs. 9-13. Meroplius cordylophorus Hennig, $\mathrm{O}^{7}$ (9-10) and Meroplius zambianus Ozerov, $0^{7}(11-13)$ : 9 - fore femur and tibia, anteriorly; 10 - end of abdomen, laterally; 11 - sternite 4; 12, — epandrium, cerci and surstyli, lateral view; 8 — same, dorsal view. 9, 10 - after Hennig [1954]; 11-13 — after Ozerov [2011].

Рис. 5-8. 9-13. Meroplius cordylophorus Hennig, О7 (9-10) and Meroplius zambianus Ozerov, О (11-13): 9 - бедро и голень передней ноги, спереди; 10 - конец брюшка, сбоку; 11 - стернит 4; 12 - эпандрий, церки и сурстили, сбоку; 13 - то же, сверху. 9, 10 - по Hennig [1954]; 11-13 - по Ozerov [2011].

DISTRIBUTION. Democratic Republic of the Congo, Ethiopia, Kenya, Republic of South Africa, Swaziland, Zimbabwe.

\section{Paratoxopoda depilis (Walker, 1849)}

MATERIAL. Malawi, Viphya Mts., Mzimba, $10 \mathrm{~km} \mathrm{SE,}$ $11^{\circ} 50.7^{\prime} \mathrm{S} 33^{\circ} 40.5^{\prime} \mathrm{E}, 1402 \mathrm{~m}, 25 . X I I .2009$, L. Friedman (1 9 , TAU); RSA, KZN, Tembe Elephant Park at: $26^{\circ} 57.963^{\prime} \mathrm{S} 32^{\circ}$ 30.707'E, 5.XII.2009, A.H. Kirk-Spriggs, ex dung of African Bush Elephant (Loxodonta africana) (2 $\left.\mathrm{O}^{7} \mathrm{O}^{7}, \mathrm{NMB}\right)$.

DISTRIBUTION. Angola, Botswana, Democratic Republic of the Congo, Ethiopia, Ivory Coast, Kenya, Malawi, Namibia, Republic of South Africa, Sierra Leone, Tanzania, Zimbabwe.

\section{Paratoxopoda mystacea sp.n.}

Figs 17-19.

MATERIAL. Holotype $0^{7}$, Central African Republic, $60 \mathrm{~km}$ W Bouar, $05^{\circ} 45^{\prime} \mathrm{N} 15^{\circ} 13^{\prime} \mathrm{E}, 660 \mathrm{~m}$, 23.III.2010, J. Halada (1 O', CULS); Paratype $\sigma^{7}$, same label as holotype (ZMUM).

DESCRIPTION. MALE. Body length 5.2-5.9 $\mathrm{mm}$; wing length 3.2-3.6 mm. Head. Frons black, subshining. Face and gena dark brown. Genal width less than width of postpedicel. Postcranium black, delicately greyish dusted. 1 small orbital, 1 ocellar, 1 divergent postocellar, 1 inner vertical setae present. Vibrissal angle with numerous short setae (Fig. 19). Antenna black. Postpedicel about 2 times as long as wide. 
Thorax black, with hardly noticeable dark blue tinge. 2 notopleural, $0+1$ supra-alar, 1 postalar, $0+1$ dorsocentral setae present. Proepisternum with several hairs in lower part. Anepisternum with one long seta near posterior margin. Postmetacoxal bridge present. Scutellum with a pair of long apical setae and a pair of lateral setulae.

Legs black. Fore coxa with strong one anteroventral seta near apex. Posterior surface of middle trochanter bare. Middle tibia with one anteroventral seta in apical quarter. Ventral surface of hind trochanter without short erect hairs.

Wing hyaline, with brown veins. Calypters whitish; margin of upper calypter white, margin of lower calypter darkened. Halter whitish.

Abdomen black, with hardly noticeable dark blue tinge, without striking setae. Posterolateral margin of tergite 5 with several fine hairs. Epandrium and surstyli as in Figs. 17, 18.

FEMALE unknown.

COMPARISON. The new species is close to Paratoxopoda crassijorceps Duda, 1926, P. barbata Ozerov, 1993, and P. glabra Ozerov, 1993 by structure of male genitalia, but all those species have two dorsocentral setae.

DISTRIBUTION. Central African Republic.

\section{Paratoxopoda pilifemorata Soós, 1964}

MATERIAL. Namibia, Katima Mulilo Dist., Ndopu village, Bukalo, near $17^{\circ} 43.660^{\prime} \mathrm{S} 2^{\circ} 4^{\circ} 32.106^{\prime} \mathrm{E}, 20-23 . I I .2012$, $900 \mathrm{~m}$, A.H. Kirk-Spriggs, malaise traps, degraded sand forest \& cultivated plots ( $\left.\sigma^{7} \sigma^{7}, \mathrm{NMB}\right)$; RSA, KZN, Tembe Elephant Park at: $26^{\circ} 57.963^{\prime} \mathrm{S} 32^{\circ} 30.707^{\prime} \mathrm{E}$, 5.XII.2009, A.H. KirkSpriggs, ex dung of African Bush Elephant (Loxodonta africana) (7 $\left.0^{7} \sigma^{7}, \mathrm{NMB}\right)$.

DISTRIBUTION. Namibia, Republic of South Africa (first record), Tanzania, Uganda.

\section{Paratoxopoda similis Ozerov, 1993}

MATERIAL. Central African Republic, $60 \mathrm{~km}$ W Bouar, $05^{\circ} 45^{\prime} \mathrm{N} 15^{\circ} 13^{\prime} \mathrm{E}, 660 \mathrm{~m}, 23 . \mathrm{III} .2010$, J. Halada (1 $\mathrm{O}^{\top}$, CULS); 60 km W Bouar, $05^{\circ} 42^{\prime} \mathrm{N} 16^{\circ} 00^{\prime} \mathrm{E}, 860 \mathrm{~m}, 30 . \mathrm{III} .2010$, J. Halada (1 $\mathrm{O}^{7}$, CULS); D.R. Congo, Oriental Province, Lieki village area at: $00^{\circ} 41.117^{\prime} \mathrm{N} 2^{\circ} 14.362^{\prime} \mathrm{E}, 25 . \mathrm{V}$.-4.IV.2010, A.H. Kirk-Spriggs, sweeping bush paths \& village environs, Boyekoli Ebale Congo Expedition 2010 ( $1 \mathrm{O}^{7}$, NMB); Nigeria, $28 \mathrm{~km}$ SEE Abuja, $09^{\circ} 06^{\prime} \mathrm{N}$ $07^{\circ} 45^{\prime} \mathrm{E}, 400 \mathrm{~m}$, 14.IV.2011, J. Halada (1 9 , CULS); Gashaka Gumfi NP, $30 \mathrm{~km} \mathrm{SE} \mathrm{Senti,} 7^{\circ} 21^{\prime} \mathrm{N} 11^{\circ} 32^{\prime} \mathrm{E}, 380-560 \mathrm{~m}$, 24.IV.8.V.2011, J. Halada (1 $\sigma^{7}, 2$ of , CULS); Togo, Kloto Agome-Yoh, $6^{\circ} 57^{\prime} \mathrm{N} 0^{\circ} 36$ 'E, $333 \mathrm{~m}$, 21.I.2011, A. Freidberg (1 $0^{7}$, TAU); Kloto, Kouma Konda, 6 ${ }^{\circ} 57^{\prime} \mathrm{N} 0^{\circ} 35^{\prime} \mathrm{E}, 620 \mathrm{~m}, 20-22 . I .2011$, A. Freidberg $(1$ +, TAU $)$.

DISTRIBUTION. Central African Republic (first record), Democratic Republic of the Congo, Ethiopia, Nigeria (first record), Togo (first record).

\section{Saltella bezzii (Duda, 1926)}

MATERIAL. Central African Republic, $45 \mathrm{~km}$ E of Nola, $3^{\circ} 40^{\prime} \mathrm{N} 16^{\circ} 20^{\prime} \mathrm{E}, 580 \mathrm{~m}, 10 . \mathrm{XI} .2012$, J. Halada $\left(2 \mathrm{O}^{7} \mathrm{O}^{7}, 4\right.$ 우,

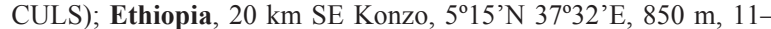
13.V.2015, J. Halada ( 2 +, CULS); Wachile env., $4^{\circ} 32^{\prime} \mathrm{N} 39^{\circ} 03^{\prime} \mathrm{E}$,
1070 m, 22.V.2015, J. Halada (1 9 , CULS); RSA, KZN, Ndumo Game R. at: $26^{\circ} 53.597^{\prime} \mathrm{S} 32^{\circ} 12.559^{\prime} \mathrm{E}, 27 . X I .2009$, A.H. KirkSpriggs, ex dung of Cape Buffalo (Syncerus caffer) (1 $0^{7}, 2$ 우, NMB); Free State, Brandfort Florisbad Res. Stat., 28 $8^{\circ} 46.039^{\prime}$ 'S $26^{\circ} 04.234^{\prime}$ E, 17-20.IX.2012, A.H. Kirk-Spriggs, malaise traps, Acacia savanna (1,$+ \mathrm{NMB})$.

DISTRIBUTION. Central African Republic (first record), Eritrea, Ethiopia (first record), Madagascar, Namibia, Nigeria, Republic of South Africa, Sudan.

\section{Sepsis arotrolabis Duda, 1926}

MATERIAL. Malawi, Nyika National Park forest, $15 \mathrm{~km} \mathrm{~N}$ Chelinda, $10^{\circ} 30.1^{\prime}$ 'S $33^{\circ} 48.8^{\prime}$ E, 2368 m, 29.XII.2009, A. Freidberg $\left(2 O^{7} O^{7}, 1\right.$, TAU); RSA, Free State, Harrismith Scotland farm at: $27^{\circ} 58^{\prime} 59.5^{\prime}$ 'S 293'09.8'E, 10-12.XI.2009, A.H. Kirk-Spriggs, malaise traps, dense Leucosedea dominated scrub $\left(30^{7} 0^{7}, 17\right.$ OP, NMB); Free State, Harrismith Geluksdal farm at: $27^{\circ} 54^{\prime} 7.05^{\prime} \mathrm{S}$ 29'23'31.9''E, 9-10.XI.2009, A.H. Kirk-Spriggs, malaise traps, sparse Leucosedea dominated scrub (2 $\left.0^{7} 0^{7}, \mathrm{NMB}\right) ; \mathrm{KZN}$, Royal Natal N.P., Thendele, $28^{\circ} 42.378^{\prime}$ 'S $28^{\circ} 56.083^{\prime}$ E, 15-17.II.2010, 1600 m, A.H. Kirk-Spriggs, malaise traps, Leucosedea dominated scrub (6 $0^{\top} 0^{\top}, 11$ 우, NMB); Free State, Harrismith Mooihoekkop, $28^{\circ} 10^{\prime} 50.0^{\prime \prime} \mathrm{S} 29^{\circ} 10^{\prime} 51.1$ ' $\mathrm{E}, 14-16 . I X .2009$, ca. $1800 \mathrm{~m}$, A.H. Kirk-Spriggs, malaise traps, Leucosedea dominated scrub (3 $\mathrm{O}^{7} \mathrm{O}^{\mathrm{T}}$, 4 9 , NMB); KZN, Royal Natal N.P., Tiger Falls area at: $28^{\circ} 41.341^{\prime} \mathrm{S} 28^{\circ} 56.047^{\prime} \mathrm{E}, 17-18 . I I .2010,1545$ m, A.H. KirkSpriggs, malaise traps, Protea caffra woodland (1 $\sigma^{\prime}, 1$, NMB); Eastern Cape, Benebos forest, $33^{\circ} 64.426^{\prime} \mathrm{S} 23^{\circ} 42.439^{\prime} \mathrm{E}, 28-$ 30.III.2009, 800 m, A.H. Kirk-Spriggs, sweeping indigenous montane forest ( 1 \% , NMB); Western Cape, Keurbos forest, $33^{\circ} 54.435^{\prime} \mathrm{S}$ $23^{\circ} 43.714^{\prime}$ E, 28-30.III.2009, 500 m, A.H. Kirk-Spriggs \& S. Otto, sweeping indigenous montane forest ( 1 , NMB); Western Cape, Gamkaskloof (Die Hel) at: $33^{\circ} 21.608^{\prime} \mathrm{S} 21^{\circ} 37.650^{\prime} \mathrm{E}, 16-18 . \mathrm{X}$. 2012, 336 m, A.H. Kirk-Spriggs, malaise traps, Karoo and valey Acacia woodland (1 $0^{7}, 1$ \% , NMB); Western Cape, Hottentots Holland N. R. at: $34^{\circ} 04.140^{\prime} \mathrm{S} 19^{\circ} 03.042^{\prime} \mathrm{E}, 11 . \mathrm{X} .2012,546 \mathrm{~m}$, A.H. Kirk-Spriggs, sweeping Pynbos and grasses (1 $\mathrm{O}^{x}$, NMB);

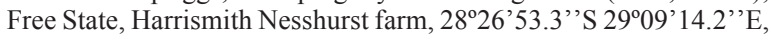
17-18.IX.2009, A.H. Kirk-Spriggs, malaise traps, Leucosedea scrub \& stream bed (1 $9, \mathrm{NMB})$; Eastern Cape, Hogsback, Redwood trail, 32³5.337's 26 $56.135^{\prime}$ E, 7.IV.2010, 1169 m, A.H. Kirk-Spriggs, sweeping indigenous Afromontane forest ( $1 \mathrm{O}^{7}$, NMB); Free State, Harrismith Scotland farm at: $27^{\circ} 59^{\prime} 21.6^{\prime \prime}$ 'S 2937'26.7''E, 11.XI.2009, A.H. Kirk-Spriggs, sweeping hillside grasses \& other vegetation (1 $9, \mathrm{NMB})$; Free State, Harrismith Geluksdal farm at: $27^{\circ} 54^{\prime} 7.05^{\prime} \mathrm{S} 29^{\circ} 23^{\prime} 31.9^{\prime} \mathrm{E}$, 9-10.XI.2009, A.H. Kirk-Spriggs, malaise traps, sparse Leucosedea dominated scrub (1 ㅇ, NMB); OFS, Bethlehem Dist., Spelonken, $2^{\circ} 28^{\prime} \mathrm{S} 28^{\circ} 38^{\prime} \mathrm{E}$, 2.IV.1994, Ent. Dept. (1 $\sigma^{7}$, NMB); OFS, Bethlehem Dist., Za-

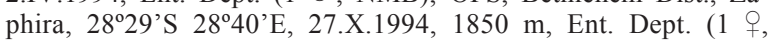
NMB).

DISTRIBUTION. Democratic Republic of the Congo, Ethiopia, Kenya, Malawi (first record), Republic of South Africa, Swaziland, Uganda.

\section{Sepsis bigemmis Duda, 1926}

MATERIAL. Cameroon, Northwest Reg., Menoua, Université Dschang, $05^{\circ} 26.761^{\prime} \mathrm{N} 10^{\circ} 04.237^{\prime} \mathrm{E}, 16 . \mathrm{VIII} .2013,1402 \mathrm{~m}$, A.H. Kirk-Spriggs, sweeping cultivated plots \& banana groves (2 $\left.\sigma^{7} \sigma^{7}, \mathrm{NMB}\right)$; Kenya, Rift Valley Prov., Emitik Lodge grounds at: $00^{\circ} 11.514^{\prime} \mathrm{N} 35^{\circ} 26.714^{\prime} \mathrm{E}, 13 . \mathrm{IV} .2011,2478 \mathrm{~m}$, A.H. \& M.K. Kirk-Spriggs, general sweeping mixed forest (1 $9, \mathrm{NMB})$; Rift Valley Prov., Njuki-Ini Forest station, $00^{\circ} 51660^{\prime} \mathrm{N} 37^{\circ} 41843^{\prime} \mathrm{E}$, 19-20.IV.2011, 1455 m, A.H. \& M.K. Kirk-Spriggs, malaise traps, remnant indigenous upland forest $(1+$, NMB).

DISTRIBUTION. Cameroon, Democratic Republic of the Congo, Kenya, Madagaskar, Nigeria, Tanzania, Uganda. 

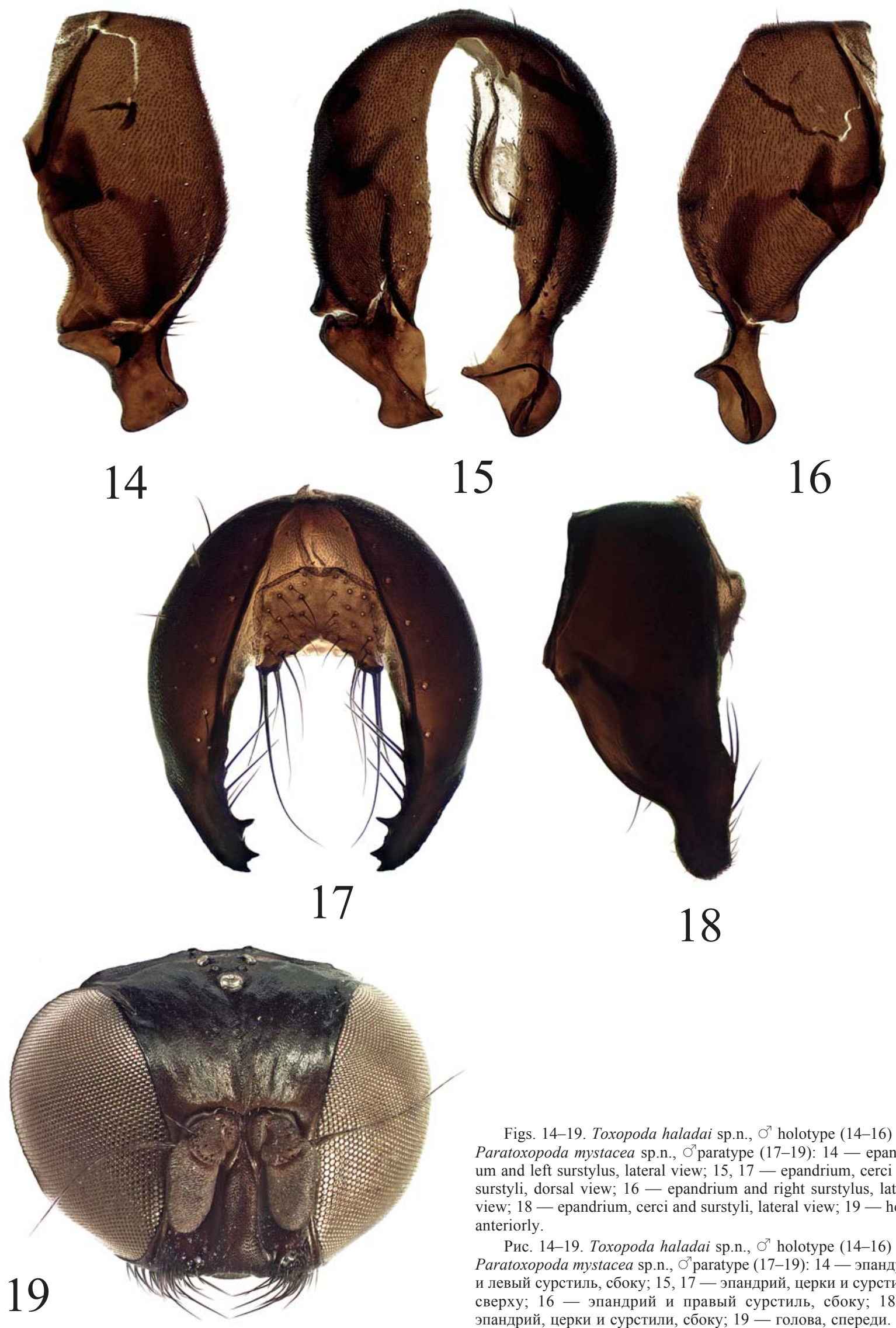

16

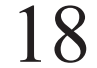

Figs. 14-19. Toxopoda haladai sp.n., O' holotype (14-16) and Paratoxopoda mystacea sp.n., O paratype (17-19): 14 - epandrium and left surstylus, lateral view; 15, 17 - epandrium, cerci and surstyli, dorsal view; 16 - epandrium and right surstylus, lateral view; 18 - epandrium, cerci and surstyli, lateral view; 19 - head, anteriorly.

Рис. 14-19. Toxopoda haladai sp.n., О7 holotype (14-16) and Paratoxopoda mystacea sp.n., O'paratype (17-19): 14 - эпандрий и левый сурстиль, сбоку; 15, 17 - эпандрий, церки и сурстили, сверху; 16 - эпандрий и правый сурстиль, сбоку; 18 эпандрий, церки и сурстили, сбоку; 19 - голова, спереди. 


\section{Sepsis delectabilis Adams, 1905}

MATERIAL. Burundi, Kayanza, Gohome, Diakotomey, $2^{\circ} 55^{\prime} \mathrm{S}$ 293'ㄹ, 1800 m, 28-29.I.2011, A. Freidberg (1 O', TAU); Cameroon, Northwest Reg., Menoua, Université Dschang, $05^{\circ} 26.761^{\prime} \mathrm{N} 10^{\circ} 04.237^{\prime} \mathrm{E}, 16 . \mathrm{VIII} .2013,1402 \mathrm{~m}$, A.H. KirkSpriggs, sweeping cultivated plots \& banana groves (1 $\left.\sigma^{7}, \mathrm{NMB}\right)$; North Reg., Vina, Tchabal village at: (1) 07³6.803'N $13^{\circ} 32.622^{\prime} \mathrm{E}$, 12.VIII.2013, 1270 m, A.H. Kirk-Spriggs, sweeping in deep shade (1 $\sigma^{7}$, NMB); D.R. Congo, Oriental Province, Likombo forest, km SW Bomane, $1.28349^{\circ} \mathrm{N} 23.72358^{\circ} \mathrm{E}, 20-22 . V .2010$, A.H. KirkSpriggs, malaise traps, lowland evergreen primary forest (disturbed), Boyekoli Ebale Congo Expedition 2010 (3 $\sigma^{7} \sigma^{7}$, NMB); Kenya, Bururi Province, Bururi Nat. Pes. cloud forest, $3^{\circ} 56^{\prime} \mathrm{S} 29^{\circ} 37^{\prime} \mathrm{E}$, 2200 m, 2.II.2011, A. Freidberg (1 $\sigma^{\top}$, TAU); Central Prov., Katura forest, Nairobi at: $01^{\circ} 14.504^{\prime} \mathrm{S} 36^{\circ} 49.452^{\prime} \mathrm{E}, 23 . \mathrm{IV} .2011,1720 \mathrm{~m}$ A.H. Kirk-Spriggs, sweeping in shaded mixed upland indigenous forest ( $\left.2 \mathrm{O}^{7} \mathrm{O}^{7}, \mathrm{NMB}\right)$; Togo, Kloto, Kouma Konda, 6 $57^{\circ} \mathrm{N} 0^{\circ} 35^{\prime} \mathrm{E}$, 620 m, 20-22.I.2011, A. Freidberg (1 O', TAU).

DISTRIBUTION. Angola, Burundi (first record), Cameroon, Democratic Republic of the Congo, Ethiopia, Kenya, Malawi, Nigeria, Republic of South Africa, Tanzania, Togo (first record), Zimbabwe, Uganda.

\section{Sepsis dissimilis Brunetti, 1910}

MATERIAL. Cameroon, Far-North Reg., Mayo-Tsanga, Mogode-Kama, $10^{\circ} 32.598^{\prime} \mathrm{N} 13^{\circ} 35.027^{\prime} \mathrm{E}, 7$.VIII.2013, $1056 \mathrm{~m}$, A.H Kirk-Spriggs,sweeping domestic donkey dung ( $2 \mathrm{O}^{7} \mathrm{O}^{7}, 3$ 우, NMB); North Reg., Vina, Tchabal village at: (1) $07^{\circ} 36.803^{\prime} \mathrm{N} 13^{\circ} 32.622^{\prime} \mathrm{E}$, 12.VIII.2013, 1270 m, A.H. Kirk-Spriggs, sweeping in deep shade (2 우, NMB); North Reg., Vina, Tchabal village at: (2) $07^{\circ} 35.195^{\prime} \mathrm{N}$ $13^{\circ} 33.566^{\prime} \mathrm{E}$, 13.VIII.2013, 1362 m, A.H. Kirk-Spriggs, malaise traps, degraded savanna hill forest, (1 $\left.\sigma^{\top}, \mathrm{NMB}\right)$; Kenya, Gilgil, 0 33'S 36² $1^{\prime}$ 'E, 9.II.2011, 1980 m, A. Freidberg (1 O, TAU); RSA, Free State, Brandfort Florisbad Res. Stat., $28^{\circ} 46.039^{\prime} \mathrm{S} 26^{\circ}$ 04.234'E, 17-20.IX.2012, A.H. Kirk-Spriggs, malaise traps, Aca cia savanna (3 $\left.\sigma^{7} \sigma^{7}, \mathrm{NMB}\right)$; Free State, Brandfort Soetdoring N.R. train camp, 2850.934'S 2601.996'E, 5-6.IV.2009, A.H. \& M.K. Kirk-Spriggs, malaise traps, Acacia savanna thicket (1 + , NMB); Free State, Harrismith Mooihoekkop, 28 10 '50.0' 'S 2910'51.1 ', E, 14-16.IX.2009, ca. 1800 m, A.H. Kirk-Spriggs, malaise traps, Leucosedea dominated scrub (1 $\sigma^{7}$, NMB); Free State, Harrismith Scotland farm at: $27^{\circ} 58^{\prime} 59.5^{\prime}$ 'S $29^{\circ} 37^{\prime} 09.8^{\prime \prime}$ E, 26-29.III.2012, A.H. Kirk-Spriggs, sweeping open grassland (4 $\sigma^{\top} \sigma^{\top}$, NMB); Free State, Petrusburg Alethiem 924 at: $28^{\circ} 42.634^{\prime} \mathrm{S} 25^{\circ} 00.882^{\prime} \mathrm{E}, 16$ 18.IV.2013, 1245 m, A.H. Kirk-Spriggs, malaise traps, Kalahari thorn veld (1 $9, \mathrm{NMB})$; Western Cape, Bloukrantz hut, $33^{\circ}$ 55'06.4' 'S 2338'17.2' 'E, 26-27.III.2009, 400 m, A.H. KirkSpriggs \& S. Otto, MV-light trap (3 $\left.\sigma^{\top} \sigma^{\top}, \mathrm{NMB}\right)$.

DISTRIBUTION. Cameroon (first record), Democratic Republic of the Congo, Ethiopia, Kenya, Madagascar, Namibia, Nigeria, Republic of South Africa, Seychelles, Swaziland, Uganda, Zimbabwe. - Australasian/Oceanian, Oriental, Palaearctic.

\section{Sepsis femoriseta Duda, 1926}

MATERIAL. Cameroon, North Reg., Vina, Tchabal village at: (1) $07^{\circ} 36.803^{\prime} \mathrm{N} 13^{\circ} 32.622^{\prime} \mathrm{E}, 12$.VIII.2013, $1270 \mathrm{~m}$, A.H. KirkSpriggs, sweeping in deep shade (1 $\sigma^{\top}, 2$ o 0 , NMB); Northwest Reg., Menoua, Université Dschang, 05²6.761'N 1004.237'E, 16.VIII.2013, 1402 m, A.H. Kirk-Spriggs, sweeping cultivated plots \& banana groves (2 90 , NMB); Far-North Reg., Mayo-Tsanga, Mogode-Kama, $10^{\circ} 32.598^{\prime} \mathrm{N} 13^{\circ} 35.027^{\prime} \mathrm{E}, 7 . \mathrm{VIII} .2013,1056 \mathrm{~m}$, A.H. Kirk-Spriggs,sweeping domestic donkey dung (1 $\sigma^{\top}, 1$, NMB); North Reg., Vina, Tchabal village at: (2) $07^{\circ} 35.195^{\prime} \mathrm{N}$ $13^{\circ} 33.566^{\prime}$ E, 13.VIII.2013, 1362 m, A.H. Kirk-Spriggs, malaise traps, degraded savanna hill forest, (1 $\left.\sigma^{\top}, \mathrm{NMB}\right)$.
DISTRIBUTION. Cameroon (first record), Ghana, Kenya, Madagascar, Namibia, Republic of South Africa, Republic of the Congo, Swaziland, Tanzania, Togo, Zimbabwe.

\section{Sepsis holaethiops Speiser, 1924}

MATERIAL. Burundi, Kayanza Province, Kiriba National Park, Rwegura, 255'S 2930'E, 2100 m, 28-30.I.2011, A. Freidberg (2 $\left.\sigma^{\top} \sigma^{\top}, \mathrm{TAU}\right)$; Kenya, Mt. Kenya, Castle Forest, 0²3'S $37^{\circ} 19^{\prime} \mathrm{E}, 1920$ and $2080 \mathrm{~m}$, 12.II.2011, A. Freidberg (5 $\sigma^{7} \sigma^{7}$, TAU); Kapkorech, 8 km NE Kericho, 0²0'S 35²1'E, 1900 m, 8.II.2011, A. Freidberg (6 $\sigma^{\top} \sigma^{\top}$, TAU); Rift Valley Prov., Timboroa Forest Station, $00^{\circ} 04.142^{\prime} \mathrm{S} 35^{\circ} 32.385^{\prime} \mathrm{E}, 14 . \mathrm{IV} .2011,2733 \mathrm{~m}$, A.H. \& M.K. Kirk-Spriggs, general sweeping mixed forest $\left(2 \bigcirc^{\top} \sigma^{\top}\right.$, NMB); Rift Valley Prov., Equator Nakuru/Eldoret rd., 0000.011'S 35³2.104'E, 13.IV.2011, 2773 m, A.H. \& M.K. Kirk-Spriggs, sweeping yellow composite and grasses ( 6 +, NMB).

DISTRIBUTION. Burundi (first record), Democratic Republic of the Congo, Kenya, Tanzania, Uganda.

\section{Sepsis igniventris Thomson, 1869}

MATERIAL. Benin, Amanhoue, Gohome, Djakotomey, 6 ${ }^{\circ} 50^{\prime} \mathrm{N}$ 146'E, 53 m, 15.I.2011, A. Freidberg (3 $\sigma^{7} \sigma^{7}$, TAU); Burundi, Kayanza, Gohome, Diakotomey, 2055'S 293'ㄹ, 1800 m, 2829.I.2011, A. Freidberg (1 $\sigma^{7}$, TAU); Cameroon, North Reg., Vina, Tchabal village at: (1) $07^{\circ} 36.803^{\prime} \mathrm{N} 13^{\circ} 32.622^{\prime} \mathrm{E}$, 12.VIII.2013, $1270 \mathrm{~m}$, A.H. Kirk-Spriggs, sweeping in deep shade (2 $\left.\sigma^{\top} \sigma^{\top}, \mathrm{NMB}\right)$; D.R. Congo, Oriental Province, Lieki village area at: $00^{\circ} 41.117^{\prime} N 24^{\circ} 14.362^{\prime} \mathrm{E}, 25$. V.-4.VI.2010, A.H. Kirk-Spriggs, malaise traps, bush paths \& village environs, Boyekoli Ebale Congo Expedition 2010 (2 $9 \circ$, NMB); Central African Republic, 60 $\mathrm{km}$ W Bouar, $05^{\circ} 42^{\prime} \mathrm{N} 16^{\circ} 00^{\prime} \mathrm{E}, 860 \mathrm{~m}$, 30.III.2010, J. Halada (2 $\sigma^{\top} \sigma^{x}$, CULS).

DISTRIBUTION. Angola, Benin (first record), Burundi (first record), Cameroon, Central African Republic (first record), Comoros, Democratic Republic of the Congo, Ethiopia, Guinea, Kenya, Madagascar, Mauritius, Nigeria, Republic of South Africa, Tanzania, Uganda, Zambia, Zimbabwe.

\section{Sepsis lateralis Wiedemann, 1830}

MATERIAL. Benin, Amanhoue, Gohome, Djakotomey, $6^{\circ} 50^{\prime} \mathrm{N} \mathrm{1} 46^{\circ} \mathrm{E}, 53 \mathrm{~m}, 15 . \mathrm{I} .2011$, A. Freidberg (4 $\sigma^{7} \mathrm{O}^{7}$, TAU);

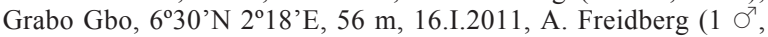
TAU); Burundi, Bujumbura Lake Tanganyika, 3'23'S $29^{\circ} 21^{\prime} \mathrm{E}$, 780 m, 27.I.2011, A. Freidberg (1 $\sigma^{7}$, TAU); Cameroon, Northwest Reg., Mezam, Bafut village at: $06^{\circ} 05.026^{\prime} \mathrm{N} 10^{\circ} 07.442^{\prime} \mathrm{E}$, 17-19.VIII.2013, 1060 m, A.H. Kirk-Spriggs, malaise traps, cultivated plots \& degraded forest (1 $\sigma^{7}$, NMB); D.R. Congo, Oriental Province, Lieki village area at: $00^{\circ} 41.117^{\prime} \mathrm{N} 24^{\circ} 14.362^{\prime} \mathrm{E}$, 25.V.-4.VI.2010, A.H. Kirk-Spriggs, malaise traps, bush paths \& village environs, Boyekoli Ebale Congo Expedition 2010 (2 $\sigma^{7} \sigma^{\top}$, 3 웅. NMB); Ethiopia, Wendo Genet, $7^{\circ} 56.799^{\prime} \mathrm{N} 38^{\circ} 36.483^{\prime} \mathrm{E}$, $1750 \mathrm{~m}$, 13.IV.2013, A. Freidberg (1 $\sigma^{7}$, TAU); Malawi, Liwonde, Shire River, Bridge, 1503.6'S 35¹3.1'E, 502 m, 10.I. 2010, A. Freidberg ( 1 T, TAU); Togo, Kloto, Kouma Konda, $6^{\circ} 57^{\prime} \mathrm{N} 0^{\circ} 35^{\prime} \mathrm{E}, 620 \mathrm{~m}, 20-22 . I .2011$, A. Freidberg (7 $\mathrm{O}^{7} \mathrm{O}^{7}, 4$ 우, TAU); Kloto waterfall, 6 $6^{\circ} 57^{\prime} \mathrm{N} 0^{\circ} 36^{\prime} \mathrm{E}, 336 \mathrm{~m}, 22 . \mathrm{I} .2011$, A. Freidberg (1 フ, TAU).

DISTRIBUTION. Angola, Benin (first record), Botswana, Burundi (first record), Cameroon, Democratic Republic of the Congo, Ethiopia, Ghana, Kenya, Madagascar, Malawi, Mauritius, Namibia, Nigeria, Republic 
of South Africa, Republic of the Congo, Réunion, Seychelles, Sierra Leone, Swaziland, Tanzania, Togo (first record), Uganda, Zambia, Zimbabwe. - Australasian/ Oceanian, Palaearctic, Oriental.

\section{Sepsis luteola Ozerov, 2004}

MATERIAL. Cameroon, North Reg., Vina, Tchabal village at: (1) $07^{\circ} 36.803^{\prime} \mathrm{N} 13^{\circ} 32.622^{\prime} \mathrm{E}, 12$. VIII.2013, $1270 \mathrm{~m}$, A.H. KirkSpriggs, sweeping in deep shade (4 $\mathrm{O}^{\top} \mathrm{O}^{\top}, 2$ 우, $\left.\mathrm{NMB}\right)$.

DISTRIBUTION. Cameroon (first record), Republic of South Africa.

\section{Sepsis macrochaetophora Duda, 1926}

MATERIAL. Cameroon, Far-North Reg., Mayo-Tsanga, Mogode-Kama, $10^{\circ} 32.598^{\prime} \mathrm{N} 13^{\circ} 35.027^{\prime} \mathrm{E}, 7 . \mathrm{VIII} .2013,1056$ m, A.H. Kirk-Spriggs, sweeping domestic donkey dung (1 $\left.\sigma^{\prime}, \mathrm{NMB}\right)$; North Reg., Vina, Tchabal village at: (1) $07^{\circ} 36.803^{\prime} \mathrm{N} 13^{\circ} 32.622^{\prime} \mathrm{E}$ 12.VIII.2013, 1270 m, A.H. Kirk-Spriggs, sweeping in deep shade (1 $\mathrm{O}^{\top}$, NMB); Northwest Reg., Menoua, Université Dschang, $05^{\circ} 26.761^{\prime} \mathrm{N} 10^{\circ} 04.237^{\prime} \mathrm{E}, 16 . \mathrm{VIII} .2013,1402 \mathrm{~m}$, A.H. Kirk Spriggs, sweeping cultivated plots \& banana groves (2 $\left.\sigma^{\top}, \mathrm{NMB}\right)$.

DISTRIBUTION. Cameroon, Democratic Republic of the Congo, Ethiopia, Guinea, Kenya, Madagascar, Republic of South Africa, Tanzania, Swaziland, Uganda. - Palaearctic (Azores).

\section{Sepsis melanota Bigot, 1886}

MATERIAL. RSA, Free State, Brandfort Florisbad Res. Stat., 2846.039'S 2604.234'E, 4-6.IV.2009, A.H. Kirk-Spriggs, malaise traps, Acacia savanna (1 + , NMB).

DISTRIBUTION. Angola, Botswana, Cameroon, Ethiopia, Kenya, Namibia, Republic of South Africa, Senegal, Zimbabwe. - Palaearctic (Egypt).

\section{Sepsis oligochaeta Soós, 1962}

MATERIAL. Cameroon, North Reg., Vina, Tchabal village at: (1) $07^{\circ} 36.803^{\prime} \mathrm{N} 13^{\circ} 32.622^{\prime} \mathrm{E}, 12 . \mathrm{VIII} .2013,1270 \mathrm{~m}$, A.H. KirkSpriggs, sweeping in deep shade $\left(8 \sigma^{7} \sigma^{7}, 7\right.$ 우, NMB); Far-North Reg., Tsanaga, Mogode at: $10^{\circ} 36.108^{\prime} \mathrm{N} 13^{\circ} 33.894^{\prime} \mathrm{E}, 7 . \mathrm{VIII} .2013$, $1015 \mathrm{~m}$, A.H. Kirk-Spriggs, sweeping cultivated plots ( 2 + $+9, \mathrm{NMB})$; RSA, Eastern Cape, Hogsback, Redwood trail, 32³5.337'S 26 56.135'E, 7.IV.2010 and 26.III.2011, 1169 m, A.H. Kirk-Spriggs, sweeping indigenous Afromontane forest ( $4 \mathrm{O}^{7} \mathrm{O}^{7}, 1$,, $\left.\mathrm{NMB}\right)$; Western Cape, Keurbos forest, 335․ $435^{\prime}$ 'S 2343.714'E, 28-30.III.2009, $500 \mathrm{~m}$, A.H. Kirk-Spriggs \& S. Otto, sweeping indigenous montane forest (28 $\sigma^{7} \sigma^{\top}, 22$ OQ, $\left.\mathrm{NMB}\right)$; Eastern Cape, Benebos forest, $33^{\circ} 64.426^{\prime}$ 'S 2342.439'E, 28-30.III.2009, 800 m, A.H. Kirk-Spriggs, sweeping indigenous montane forest ( $2 \sigma^{\top} \sigma^{\top}, 1$, , NMB); Western Cape, Buffelsbos forest, 33⒌154'S 2338.329'E, 27.III.2009, 400 m, A.H. Kirk-Spriggs, sweeping paths indigenous montane forest (1 $\sigma^{7}$, NMB); Tsitsikamma N.P., Rugbos Forest trail at: $33^{\circ} 57.563^{\prime} \mathrm{S}$ 2340.198'E, 22.I.2009, A.H. Kirk-Spriggs \& S. Otto, sweeping indigenous montane (1 + , NMB); KwaZulu-Natal, Kosi Bay Nature Res. at (1): 26057'21''S 3249'51''E, 10-13.XII.2010, 37 m, A.H. Kirk-Spriggs, malaise traps, indigenous dune forest (1\%, NMB).

DISTRIBUTION. Cameroon (first record), Kenya, Malawi, Republic of South Africa.

Sepsis pedunculata Duda, 1926

MATERIAL. RSA, Free State, Harrismith Scotland farm at: 27'58'59.5'S 29³7'09.8'E, 10-12.XI.2009, A.H. Kirk-Spriggs, ma- laise traps, dense Leucosedea dominated scrub ( $8 \sigma^{7} \sigma^{7}, 6$ 우, NMB); Free State, Harrismith Mooihoekkop, $28^{\circ} 10^{\prime} 50.0^{\prime} \mathrm{S}$ '29 $9^{\circ} 10^{\prime} 51.1^{\prime} \mathrm{E}$, 14-16.IX.2009, ca. 1800 m, A.H. Kirk-Spriggs, malaise traps, Leucosedea dominated scrub (26 $\sigma^{\top} \sigma^{\top}, 32$ 90, NMB); Free State, Harrismith Geluksdal farm at: $27^{\circ} 54^{\prime} 7.05^{\prime} \mathrm{S} 29^{\circ} 23^{\prime} 31.9^{\prime} \mathrm{E}$, 910.XI.2009, A.H. Kirk-Spriggs, malaise traps, Leucosedea dominated scrub (1 $\sigma^{\prime}$, NMB); KZN, Royal Natal N.P., Thendele, 28²42.378'S 2856.083'E, 15-17.II.2010, 1600 m, A.H. Kirk-Spriggs, malaise traps, Leucosedea dominated scrub (1 + , NMB).

DISTRIBUTION. Cameroon, Democratic Republic of the Congo, Ethiopia, Kenya, Republic of South Africa, Tanzania, Uganda.

\section{Sepsis petulantis Adams, 1905}

MATERIAL. Central African Republic, $60 \mathrm{~km}$ W Bouar, $05^{\circ} 45^{\prime} \mathrm{N} 15^{\circ} 13^{\prime} \mathrm{E}, 660 \mathrm{~m}, 23 . \mathrm{III} .2010$, J. Halada (1 $0^{7}$, CULS); Togo, Kloto, Kouma Konda, 657’'N 035'ㄹ, 620 m, 20-22.I.2011, A. Freidberg (3 O $^{7} \mathrm{O}^{7}$, TAU); Kloto Agome-Yoh, 6 $6^{\circ} 57^{\prime} \mathrm{N} 0^{\circ} 36^{\prime} \mathrm{E}$, 333 m, 21.I.2011, A. Freidberg ( $1 \mathrm{O}^{7}$, TAU).

DISTRIBUTION. Central African Republic (first record), Ethiopia, Malawi, Togo (first record), Zimbabwe.

\section{Sepsis pronodosa Speiser, 1924}

MATERIAL. Malawi, Nyika National Park forest, $15 \mathrm{~km} \mathrm{~N}$ Chelinda, $10^{\circ} 30.1^{\prime} \mathrm{S} 33^{\circ} 48.8^{\prime} \mathrm{E}, 2368 \mathrm{~m}, 29 . X I I .2009$, A. Freidberg (1 ऽ, TAU); Nyika National Park, Chelinda15 km W, $10^{\circ} 35^{\prime} \mathrm{S}$ $33^{\circ} 44.1^{\prime}$ 'E, 2234 m, 31.XII.2009, A. Freidberg (1 $\sigma^{7}$, TAU); Zomba Plateau Trout Farm, 15²1.4'S 35 $5^{\circ} 18.1^{\prime} \mathrm{E}, 1477$ m, 10.I.2010, A. Freidberg (2 $\sigma^{7} \sigma^{7}$, TAU).

DISTRIBUTION. Democratic Republic of the Congo, Kenya, Malawi, Republic of South Africa, Sudan, Tanzania, Uganda.

Sepsis sternopleuralis Duda, 1926

MATERIAL. RSA, Western Cape, Keurbos forest, $33^{\circ} 54.435^{\prime} \mathrm{S}$ $23^{\circ} 43.714^{\prime}$ E, 28-30.III.2009, 500 m, A.H. Kirk-Spriggs \& S. Otto, sweeping indigenous montane forest ( $\left.1 \sigma^{7}, \mathrm{NMB}\right)$.

DISTRIBUTION. Republic of South Africa.

\section{Sepsis thoracica Robineau-Desvoidy, 1830}

MATERIAL. Burundi, Kayanza, 2º5''S $29^{\circ} 37^{\prime} E, 1800$ m, 2829.I.2011, A. Freidberg (2 $\sigma^{\top} \sigma^{\top}$, TAU); Cameroon, Far-North Reg., Mayo-Tsanga, Mogode-Kama, $10^{\circ} 32.598^{\prime} \mathrm{N} 13^{\circ} 35.027^{\prime} \mathrm{E}$, 7.VIII.2013, 1056 m, A.H. Kirk-Spriggs,sweeping domestic donkey dung (3 $\sigma^{\top} \sigma^{\top}, 19$ \% 19 NMB); North Reg., Mayo-Louti, Bossom at: (2) $09^{\circ} 55.233^{\prime} \mathrm{N} 13^{\circ} 46.645^{\prime} \mathrm{E}, 6$.VIII.2013, 475 m, A.H. Kirk-Spriggs, sweeping understory vegetation in deep shade ( $1 \sigma^{7}, 8$ 우, NMB); Northwest Reg., Mezam, Bafut village at: 0605.026'N 1007.442'E, 17-19.VIII.2013, 1060 m, A.H. Kirk-Spriggs, malaise traps, cultivated plots \& degraded forest ( $2 \sigma^{\top} \sigma^{7}$, NMB); Northwest Reg., Mezam, Sincoa village at: $05^{\circ} 45.019^{\prime} \mathrm{N} 10^{\circ} 09.589^{\prime} \mathrm{E}, 20$.VIII.2013, 1614 m, A.H. Kirk-Spriggs, sweeping grasses \& other vegetation ( 4 $\left.{ }_{+\rightarrow}, \mathrm{NMB}\right)$; Far-North Reg., Mayo-Tsanga, Mogode-Cha, $10^{\circ} 34.111^{\prime} \mathrm{N}$ $13^{\circ} 36.422^{\prime}$ E, 8-10.VIII.2013, 1001 m, A.H. Kirk-Spriggs, malaise traps, degraded savanna forest (1 $\Im^{\top}, 2$ + 9 , NMB); North Reg., Vina, Tchabal village at: (2) $07^{\circ} 35.195^{\prime} \mathrm{N} 13^{\circ} 33.566^{\prime} \mathrm{E}, 13$.VIII.2013, 1362 m, A.H. Kirk-Spriggs, malaise traps, degraded savanna hill forest, (2 $\left.\sigma^{7} \sigma^{7}, \mathrm{NMB}\right)$; Central African Republic, $45 \mathrm{~km} \mathrm{E}$ of Nola, $3^{\circ} 40^{\prime} \mathrm{N}$ $16^{\circ} 20^{\prime} \mathrm{E}, 580 \mathrm{~m}, 10 . X \mathrm{X} .2012$, J. Halada (4 ○ $^{\top} \sigma^{\top}, 2$ 우, CULS); Namibia, prov. Erongo, Usakos, $21^{\circ} 58^{\prime}$ 'S 15³6'E, 900 m, 14.III.2014, J. Halada (1 $\sigma^{7}$, CULS); RSA, Free State, Harrismith Scotland farm at: $27^{\circ} 59^{\prime} 21.6$ ''S $29^{\circ} 37^{\prime} 26.7^{\prime}$ 'E, 11.XI.2009, A.H. Kirk-Spriggs, sweeping hillside grasses $\&$ other vegetation ( $2 \mathrm{O}^{\top} \mathrm{O}^{\top}, 4$ 우, NMB); 
Free State, Harrismith Scotland farm at: $27^{\circ} 58^{\prime} 59.5^{\prime \prime} \mathrm{S} 29^{\circ} 37^{\prime} 09.8^{\prime \prime} \mathrm{E}$, 10-12.XI.2009, A.H. Kirk-Spriggs, malaise traps, dense Leucosedea dominated scrub ( 4 +, NMB); Free State, Harrismith Geluksdal farm at: $27^{\circ} 54^{\prime} 7.05^{\prime}$ 'S 29²3'31.9''E, 9-10.XI.2009, A.H. Kirk-Spriggs, sweeping cattle/horse dung ( $\Im^{\prime}, 4$, 4 , NMB); Free State, Harrismith Mooihoekkop, 28¹0'50.0'S 29¹0'51.1'’E, 14-16.IX.2009, ca. 1800 m, A.H. Kirk-Spriggs, malaise traps, Leucosedea dominated scrub (2 $\sigma^{\top} \sigma^{\top}, 2$ 우, NMB); Free State, Brandfort Florisbad Res. Stat. $28^{\circ} 46.039^{\prime}$ 'S 2604.234'E, 17-20.IX.2012, A.H. Kirk-Spriggs, malaise traps, Acacia savanna (12 $\sigma^{\top} \sigma^{7}, 6$ 우, NMB); KZN, Royal Natal N.P., Thendele, $28^{\circ} 42.378^{\prime}$ 'S 28 $8^{\circ} 56.083^{\prime}$ E, 15-17.II.2010, $1600 \mathrm{~m}$, A.H. Kirk-Spriggs, malaise traps, Leucosedea dominated scrub (4 $\mathrm{O}^{7} \sigma^{\top}, 6$ +9, NMB); Western Cape, Bloukrantz hut, 3355'06.4' 'S 2338'17.2' E, 26-27.III.2009, 400 m, A.H. Kirk-Spriggs \& S. Otto, MV-light trap (1 $9, \mathrm{NMB})$; Western Cape, R328 near Matjiesriver, 33⒉363'S $21^{\circ} 59.538^{\prime}$ E, 15.X.2012, 672 m, A.H. Kirk-Spriggs, sweeping roadside vegetation ( 3 +, NMB); Eastern Cape, Baviaanskloof Nat. Res., Berglaas trail hut at: 3338.075'S 2429.306'E, 28.I.2009, A.H. Kirk-Spriggs \& S. Otto, sweeping grassy Fynbos (1 $\sigma^{\top}$, NMB); Free State, Harrismith Scotland farm at: $27^{\circ} 58^{\prime} 59.5^{\prime \prime} \mathrm{S} 29^{\circ} 37^{\prime} 09.8^{\prime}$ 'E, 26-29.III.2012, A.H. Kirk-Spriggs, sweeping open grassland (1 $\sigma^{7}$ NMB); OFS, Bethlehem Dist. Avondrust, $28^{\circ} 28^{\prime} \mathrm{S} 28^{\circ} 41^{\prime} \mathrm{E}, 24$ 27.X.1994, Ent. Dept. (1 $9, \mathrm{NMB})$.

DISTRIBUTION. Burundi (first record), Cameroon (first record), Central African Republic (first record), Democratic Republic of the Congo, Ethiopia, Kenya, Namibia, Nigeria, Republic of South Africa, Swaziland, Uganda, Yemen, Zambia, Zimbabwe. - Australasian/ Oceanian, Oriental, Palaearctic.

\section{Sepsis validiseta Duda, 1926}

MATERIAL. Kenya, Mt. Kenya, Niukiini Forest, swamp, $0^{\circ} 31$ 'S $37^{\circ} 25^{\prime}$ E, 13.II.2011, A. Freidberg (1 ऽ', TAU); RSA, KZN, Royal Natal N.P., Thendele, 2842.378'S $28^{\circ} 56.083^{\prime}$ 'E, 15-17.II.2010, 1600 m, A.H. Kirk-Spriggs, malaise traps, Leucosedea dominated scrub

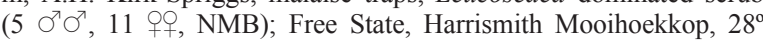
10'50.0'’S 2910'51.1''E, 14-16.IX.2009, ca. 1800 m, A.H. KirkSpriggs, malaise traps, Leucosedea dominated scrub $\left(3 \sigma^{\top} \sigma^{\top}, 1\right.$, NMB); Free State, Brandfort Florisbad Res. Stat., 2846.039'S $26^{\circ} 04.234^{\prime}$ 'E, 17-20.IX.2012, A.H. Kirk-Spriggs, malaise traps, Acacia savanna (1 $\sigma^{7}, 1$ q, NMB); Western Cape, Anysberg Nature Reserve at: $33^{\circ} 27.829^{\prime} \mathrm{S} 20^{\circ} 35.456^{\prime} \mathrm{E}, 13-14 . X .2012,747$ m, A.H Kirk-Spriggs, malaise traps, Karoo vegetation ( $\left.1 \sigma^{7}, \mathrm{NMB}\right)$; Eastern Cape, Baviaanskloof Nat. Res., Berglaas trail hut at: $33^{\circ} 38.075^{\prime} \mathrm{S}$ 24²9.306'E, 28.I.2009, A.H. Kirk-Spriggs \& S. Otto, sweeping grassy Fynbos ( $1+$ NMB); Free State, Harrismith Scotland farm at: $27^{\circ} 58^{\prime} 59.5^{\prime}$ 'S 29³7'09.8''E, 10-12.XI.2009, A.H. Kirk-Spriggs, malaise traps, dense Leucosedea dominated scrub (1 $\left.\sigma^{7}, \mathrm{NMB}\right)$; Free State, Harrismith Nesshurst farm, 28 26'53.3'’S 2909'14.2' 'E, 17 18.IX.2009, A.H. Kirk-Spriggs, malaise traps, Leucosedea -scrub \& stream bed $(1+\mathrm{N}, \mathrm{NMB})$.

DISTRIBUTION. Cameroon, Democratic Republic of the Congo, Ethiopia, Guinea, Kenya, Malawi, Republic of South Africa, Swaziland, Tanzania.

Toxopoda au Ozerov, 1998

MATERIAL. Namibia, Katima Mulilo Dist., Mudumu N.P. Nakatwa at: $18^{\circ} 10.254^{\prime} \mathrm{S} 23^{\circ} 25.183^{\prime} \mathrm{E}, 26-27 . X I .2012,952 \mathrm{~m}$, A.H. Kirk-Spriggs, malaise traps, Miombo \& Mopane Woodlands $\left(1 \sigma^{7}, \mathrm{NMB}\right)$.

DISTRIBUTION. Angola, Botswana, Kenya, Namibia, Republic of South Africa.

\section{Toxopoda bequaerti (Curran, 1929)}

MATERIAL. Burundi, Muyinga Province, Ruvubu National Park, savanna, $2^{\circ} 54^{\prime} \mathrm{S} 30^{\circ} 22^{\prime} \mathrm{E}, 1380 \mathrm{~m}$, 30.I.2011, A. Freidberg (2 $\sigma^{\top} \sigma^{\top}, 2$
$9 \circ$, TAU); Central African Republic, $60 \mathrm{~km} \mathrm{~W}$ Bouar, $05^{\circ} 45^{\prime} \mathrm{N}$ $15^{\circ} 13^{\prime} \mathrm{E}, 660 \mathrm{~m}$, 23.III.2010, J. Halada (1 $\mathrm{O}^{7}$, CULS); $60 \mathrm{~km}$ W Bouar, $05^{\circ} 42^{\prime} \mathrm{N} 16^{\circ} 00^{\prime} \mathrm{E}, 860 \mathrm{~m}$, 30.III.2010, J. Halada (1 $\sigma^{7}$, CULS).

DISTRIBUTION. Burundi (first record), Cameroon, Central African Republic (first record), Democratic Republic of the Congo, Malawi, Namibia, Republic of South Africa, Zimbabwe.

\section{Toxopoda haladai sp.n. Figs 14-16.}

MATERIAL. Holotype $\sigma^{7}$, Central African Republic, $60 \mathrm{~km}$

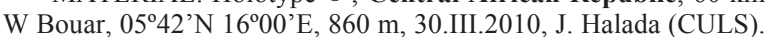

DESCRIPTION. MALE. Body length $4.5 \mathrm{~mm}$; wing length $2.9 \mathrm{~mm}$. Head flattened dorsoventrally. Eye in profile roundish. Frons black, only reddish near anterior margin, matt. Face and gena light brown. Lower margin of face protruding anteriorly. Postcranium black, delicately greyish dusted. 1 orbital, 1 ocellar, 1 postocellar, 1 outer vertical setae present. Antenna light brown. Postpedicel in lateral view is apically narrowing, approximately 2.5 times as long as wide near base. One vibrissa present.

Thorax black, delicately greyish dusted. 1 postpronotal, 2 notopleural, $0+1$ supra-alar, 1 postalar, $0+2$ dorsocentral setae present. Proepisternum with several hairs near lower margin. Anepisternum with one long seta near posterior margin. Katepisternum delicately greyish dusted completely. Anepimeron bare, without hairs. Postmetacoxal bridge present. Scutellum with a pair of long apical setae.

Legs black. Fore coxa with strong one anteroventral seta near apex. Fore femur with two rows of spines ventrally. Mid femur curved at middle. Mid tibia with a row of short posteroventral setae. Hind femur with a row of dorsal setae in apical half. Hind tibia with 3-4 dorsal setae.

Wing tinged with brownish, with brown veins. Calypters whitish; margin of upper calypter white, margin of lower calypter darkened. Halter blackish.

Abdomen black, without strong setae. Epandrium and surstyli as in Figs. 14-16. Surstyli asymmetrical, not fused to epandrium.

FEMALE unknown.

COMPARISON. The new species has katepisternum delicately greyish dusted completely and asymmetrical surstyli as in Toxopoda bequaerti Curran, 1929, T. vanschuytbroecki Ozerov, 1993, T. soror Munari, 1994, and T. au Ozerov, 1998. The new spesies is close to Toxopoda vanschuytbroecki and T. au by structure of right surstylus, but differs clearly by structure of left surstylus.

ETYMOLOGY. The species is named after Czech entomologist Mr. Jiri Halada, the collector of the holotype specimen.

\section{DISTRIBUTION. Central African Republic.}

\section{Toxopoda nitida Macquart, 1851}

MATERIAL. Cameroon, Far-North Reg., Mayo-Tsanga, Mogode-Cha, $10^{\circ} 34.111^{\prime} \mathrm{N} 13^{\circ} 36.422^{\prime} \mathrm{E}, 8^{8-10 . V I I I .2013, ~} 1001 \mathrm{~m}$, A.H. 
Kirk-Spriggs, malaise traps, degraded savanna forest $(1 \stackrel{\circ}{\circ}, \mathrm{NMB})$; Namibia, Katima Mulilo Dist., Mavunje campsite at: $17^{\circ} 55.141^{\prime}$ 'S $23^{\circ} 19.073^{\prime}$ E, 22-26.XI.2012, 945 m, A.H. Kirk-Spriggs, malaise traps, Kwando River floodplain (1 $\mathrm{O}^{7}$, NMB); Katima Mulilo Dist., Mudumu N.P. Nakatwa at: $18^{\circ} 10.254^{\prime} S 23^{\circ} 25.183^{\prime} E, 26-$ 27.XI.2012, 952 m, A.H. Kirk-Spriggs, malaise traps, Miombo \& Mopane Woodlands ( 1 , NMB); RSA, KZN, Ndumo Game R. pan at: $26^{\circ} 54.288^{\prime} \mathrm{S} 32^{\circ} 17.974^{\prime} \mathrm{E}$, 9-10.XII.2009, A.H. Kirk-Spriggs, malaise traps, grassy floodplain (2 $90, \mathrm{NMB})$; Zambia, Mumbwa Dist., Kafue National Park, Mayukuyuku camp area at: $14^{\circ} 54.898^{\prime}$ 'S $26^{\circ} 03.820^{\prime}$ E, 3-5.XII.2012, 1081 m, A.H. \& M.K. Kirk-Spriggs, malaise traps, Central Zambezian Miombo Woodlands (1 $\left.0^{7}, \mathrm{NMB}\right)$.

DISTRIBUTION. Angola, Botswana, Cameroon (first record), Madagascar, Malawi, Namibia, Republic of South Africa, Republic of the Congo, Sudan, Zambia. - Palaearctic (Egypt).

Toxopoda soror (Munari, 1994)

MATERIAL. Central African Republic, $60 \mathrm{~km}$ W Bouar, $05^{\circ} 45^{\prime} \mathrm{N} 15^{\circ} 13^{\prime} \mathrm{E}, 660 \mathrm{~m}$, 23.III.2010, J. Halada (1 $0^{\top}$, CULS);

DISTRIBUTION. Central African Republic (first record), Democratic Republic of the Congo, Sierra Leone.

ACKNOWLEDGEMENTS. The work was conducted within the state project No AAAA-A16-116021660077-3. I am very grateful to Drs. Miroslav Barták (CULS), Amnon
Freidberg (TAU) and Ashley Kirk-Spriggs (NMB) for the material of Sepsidae for study.

\section{References}

Cumming J.M., Wood D.M. 2009. Adult morphology and terminology // Brown B.V., Borkent A., Cumming J.M., Wood D.M., Woodley N.E., Zumbado M. (eds.). Manual of Central American Diptera. Vol.1. Ottawa: National Research Council Press. P.9-50.

Hennig W. 1954. Piophilidae und Sepsidae aus Ost-Afrika (Diptera). // Beiträge zur Entomologie. Bd.4. Nr.5/6. S.641-643.

McAlpine J.F. 1981. Morphology and terminology-adults // McAlpine J.F., Peterson B.V., Shewell G.E., Teskey H.J., Vokeroth J.R., Wood D.M. (coord.). Manual of Nearctic Diptera. Vol.2. Ottawa: Research Branch. Agriculture Canada. Monograph 27. P.9-63.

Ozerov A.L. 2005. World Catalogue of the family Sepsidae (Insecta: Diptera) // Zoologicheskie Issledovania. Moscow. Vol.8. 74 p.

Ozerov A.L. 2006. Studies of Afrotropical Sepsidae (Diptera). V. Key and diagnoses of genera of Afrotropical Sepsidae // Russian Entomological Journal. Vol.15. No.1. P.93-108.

Ozerov A.L. 2010. Three new species of Sepsidae (Diptera) // Far Eastern Entomologist. No.209. P.1-6.

Ozerov A.L. 2011. A new species of Meroplius Rondani (Diptera, Sepsidae) from Zambia // Far Eastern Entomologist. No.229. P.11-12.

Stuckenberg B.R. 1999. Antennal evolution in the Brachycera (Diptera), with a reassessment of terminology relating to the flagellum // Studia Dipterologica. Vol.6. S.33-48. 